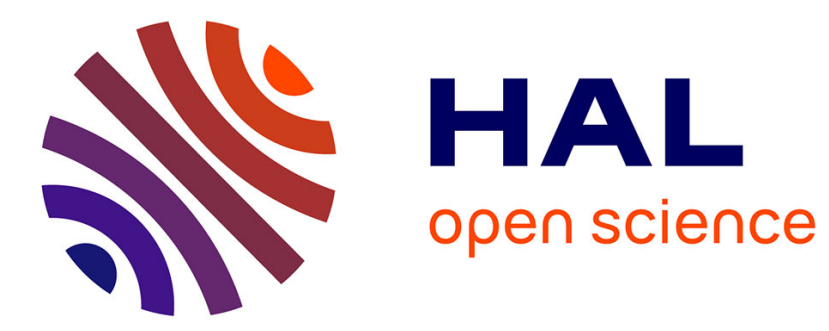

\title{
Cartographier la trajectoire de l'adaptation dans l'espace des négociations sur le climat
}

Nicolas Baya-Laffite, Jean-Philippe Cointet

\section{To cite this version:}

Nicolas Baya-Laffite, Jean-Philippe Cointet. Cartographier la trajectoire de l'adaptation dans l'espace des négociations sur le climat: Changer d'échelle, réd(u)ire la complexité. Réseaux: communication, technologie, société, 2014, 6 (188), pp.159-198. 10.3917/res.188.0159 . hal-01128560

\section{HAL Id: hal-01128560 \\ https://hal.science/hal-01128560}

Submitted on 9 Mar 2017

HAL is a multi-disciplinary open access archive for the deposit and dissemination of scientific research documents, whether they are published or not. The documents may come from teaching and research institutions in France or abroad, or from public or private research centers.
L'archive ouverte pluridisciplinaire HAL, est destinée au dépôt et à la diffusion de documents scientifiques de niveau recherche, publiés ou non, émanant des établissements d'enseignement et de recherche français ou étrangers, des laboratoires publics ou privés. 


\title{
CARTOGRAPHIER LA TRAJECTOIRE DE L'ADAPTATION DANS L'ESPACE DES NEGOCIATIONS SUR LE CLIMAT : CHANGER D'ECHELLE, RED(U)IRE LA COMPLEXITE
}

\author{
NICOLAS BAYA-LAFFITE ${ }^{12}$ et JEAN-PHILIPPE \\ COINTET $^{34}$
}

\footnotetext{
${ }^{1}$ Auteur correspondant: Nicolas Baya-Laffite, medialab (EA7033), Sciences Po, 27 rue Saint Guillaume, 75337 Cedex 07, Paris 75337, France. Email: nicolas.bayalaffite@sciencespo.fr.

${ }^{2}$ MEDIALAB SCIENCES-PO

${ }^{3}$ LISIS, CORTEXT, IFRIS

${ }^{4}$ Nicolas Baya Laffite a rédigé la version originale du manuscrit; Jean-Philippe Cointet, a contribué au design et à la mise en oeuvre du protocole expérimental et à la précision des aspects méthodologiques du manuscrit. Les résultats ici présentés découlent d'une expérience collective où ont participé, outre les deux auteurs de cet article : Tommaso Venturini, Ian Gray, Benjamin Ooghe-Tabanou, Kari de Pryck (médialab Sciences Po), et Vinciane Zabban (Université Paris 13). Ont collaboré, également Martina Cecchi et Paolo Ciuccarelli (Density Design), partenaires $\mathrm{du}$ médialab daans le cadre $\mathrm{du}$ projet EMAPS (http://www.emapsproject.com/blog/).
} 
Résumé : Les négociations qui se tiennent depuis vingt ans au sein de Convention cadre des Nations Unies sur les changements climatiques (CCNUCC) à l'aune de l'objectif d'atténuation des causes du réchauffement climatique connaissaient une inflation thématique incessante à l'aune des intérêts et cadrages souvent concurrents des Parties. En témoigne la place acquise par le thème, longtemps controversé, de l'adaptation aux impacts du changement climatique. Cartographier ce phénomène complexe constitue un défi pour l'analyse quali-quantitative des problèmes publics que cet article adresse en présentant et en discutant une expérience de production et de visualisation de données sur la configuration de l'espace thématique des négociations et son déploiement dans le temps. Le changement d'échelle ainsi opéré permet de réduire et redire la complexité pour rendre compte, entre visualisation et narration, des évolutions croisées des notions d'atténuation et d'adaptation tout le long du processus de négociation. Celui-ci n'est, cependant, qu'un exemple parmi les multiples histoires possibles qui pourraient être produites. Ainsi, ce type de modèle et de visualisation porte un potentiel en tant qu'objet frontière pour discuter les enjeux du présent ou du futur à l'aune d'une vue synoptique du processus.

Le processus international de négociation sur le climat se caractérise par une complexité toujours croissante. Deux facteurs au moins en sont à l'origine. Le premier a trait à sa durée, sa grammaire et sa portée universelle (Gemenne, 2013b). Trois dates clés servent de repère pour identifier les origines de la politique climatique internationale: 1979 d'abord, avec l'identification du problème climatique mondiale lors de la première conférence sur le climat tenue à Genève ; 1988 ensuite, avec la création le Groupe intergouvernemental d'experts sur le climat (GIEC) par l'Organisation météorologique mondiale et le programme de nations unies pour l'environnement; et 1990 enfin, avec la publication du premier rapport d'évaluation du GIEC et l'organisation de la seconde conférence mondiale sur le climat. C'est à l'issue de celle-ci que fut décidée la création d'un instrument de politiques internationales: la Convention cadre des Nations Unies sur les changements climatiques (CCNUCC). Négociée pendant deux ans, et adoptée à la veille de du Sommet de Rio sur l'environnement et le développement de 1992, la CCNUCC est à la fois le principal instrument juridique et arène politique du régime de gouvernance climatique (le GIEC incarnant l'arène de l'expertise climatique). C'est à l'occasion des sessions de l'organe suprême de la Convention, la Conférence des Parties (COP), tenues chaque année depuis 1995 dans une ville différente, que les délégations représentant maintenant 196 parties (y compris l'Union Européenne), se réunissent, accompagnées d'une grande variété d'acteurs non étatiques, pour gouverner un problème posé 
essentiellement en termes mondiaux (Aykut, 2012). Le but des sessions de la COP est de négocier, décider et instrumenter la mise en œuvre de l'objectif ultime de la Convention, à savoir, «stabiliser [...] les concentrations de gaz à effet de serre (GES) dans l'atmosphère à un niveau qui empêche toute perturbation anthropique dangereuse du système climatique ». Et ce, précise le texte, «dans un délai suffisant pour que les écosystèmes puissent s'adapter naturellement aux changements climatiques, que la production alimentaire ne soit pas menacée et que le développement économique puisse se poursuivre d'une manière durable $»^{5}$. Cet objectif d'atténuation des causes du changement climatique est recherché à l'aune des principes du droit onusien de la coopération multilatérale en matière d'environnement. Parmi ceux-ci, les responsabilités communes mais différenciées, fondé sur la responsabilité historique du problème des pays développés et donnant lieu à un partage équitable $\mathrm{du}$ fardeau: d'une part, la définition d'engagements contraignants de réduction des émissions de GES distinguant entre pays en développement (PED) et pays développés en fonction de leurs capacités respectives; d'autre part, la mise en place de mécanismes d'aide au transfert de technologies et au financement de la lutte contre le changement climatique des PED. Les engagements de réduction ne valant que pour des périodes déterminées, les Parties, regroupées dans des coalitions d'intérêts diverses, variables et chevauchées, doivent les renouveler. Et ce, à l'aune de réalités nouvelles qui, depuis 2007, conduisent à une discussion des fondements normatifs de la négociation. C'est donc là un processus permanent de portée universelle, dont rien, malgré ses échecs répétés, ne semble indiquer pour l'heure qu'il doive bientôt s'interrompre.

Dans ces circonstances, l'agenda des COP connaît une l'inflation thématique concomitante qui conduit à une parcellisation des négociations en sous-processus disparates, toujours plus techniques et spécifiques (Aykut et Dahan, 2015). C'est là un second facteur de complexité. Celui-ci a trait à l'évolution constante des connaissances sur le problème et des enjeux géopolitiques relatifs à la manière de s'y s'attaquer. D'une part, les connaissances climatiques, tout comme le problème qu'elles adressent, n'ont pas cessé de gagner du terrain depuis vingt ans. Elles acquièrent par là une transversalité politique croissante. En témoigne l'évolution des rapports d'évaluation que le GIEC publie tous les 5-7 ans environ depuis 1990 comme une base de connaissances factuelles constamment actualisée. Evolution où le volet «impacts, vulnérabilité et adaptation» se voit accorder une importance accrue à l'aune des avancées de la modélisation du système Terre (Dahan, 2010), mais aussi des contributions en sciences humaines et sociales à la compréhension du problème. Ce sont là des évolutions qui jalonnent le processus de négociation. D'autre part, les COP fonctionnent comme un forum de négociation entre les intérêts souvent concurrents du Nord et du Sud; des intérêts qui touchent à une trop vaste gamme de questions, liées mais distinctes, de celle de la définition des engagements de réductions de GES. Les questions de développement et de pauvreté qui sont intrinsèquement liées à celles de vulnérabilité au aux impacts du changement climatique et, partant, de l'adaptation par l'anticipation y sont centrales. Adaptation et atténuation sont les deux grandes stratégies pour gérer les risques d'un changement climatique d'origine anthropique rapide et en constante évolution; des risques qui résultent de l'interaction constante entre ces changements du système climatique et des changements des sociétés humaines. A la différence des

\footnotetext{
${ }^{5}$ Art. 2 de la CCNUCC.
} 
politiques d'atténuation s'attaquant aux causes du changement climatique pour limiter les perturbations humaines sur le système climatique, les politiques d'adaptation se focalisent sur la réduction des conséquences des impacts du changement climatique (incendies de forêts, inondations, manque d'eau, sécheresses, érosion et submersion des côtes, modifications profondes des écosystèmes, nouvelles maladies, tempêtes extrêmes, vagues de chaleur, ouragans, fonte des glaciers), et ce en agissant sur les systèmes humains (affectés de surcroît par des changements démographiques, socioéconomiques, cultures, politiques, technologiques): d'une part, en réduisant leur vulnérabilité (c'est à dire la propension ou prédisposition à subir des dommages) face aux impacts anticipés, et d'autre part, en augmenter leur résilience ; (c'est à dire la capacité d'absorber des impacts) face aux impacts non anticipés des changements climatiques. Or, la rapidité $\mathrm{du}$ changement rend la planification et l'anticipation indispensables, une adaptation spontanée étant largement insuffisante. Et c'est tant les évolutions du climat que celles de la société que l'on doit anticiper pour s'adapter. A ceci toute cette complexité, l'origine anthropique du changement rajoute une dimension politique à l'adaptation qui se pose avec acuité au sein de la CCNUCC, à savoir celle de la responsabilité, à la base des débats sur le financement de l'adaptation et de la réparation des pertes de dommages causés par les catastrophes climatiques. La question est capitale car les pays développées, en développement et les moins développées se trouvent dans des situations très inégales en termes d'exposition, vulnérabilité et capacité d'adaptation face aux impacts d'un changement climatique, dont les responsables historiques sont les pays développés. C'est à l'aune de ces questionnements que, dans un régime d'atténuation plus marqué par ses échecs que par ses réussites, une nouvelle approche du problème climatique (Pielke Jr., 2005), poussée par les PED conduit depuis 2000 à incorporer de plus en plus cette question d'adaptation anticipatrice et planifiée comme objet de politiques et de financements. Nouvel objectif des négociations, l'impératif d'adaptation reconfigure progressivement leurs enjeux. On a ainsi parlé d'un « tournant de l'adaptation » (Dahan et Aykut, 2012); tournant qui, malgré la clôture relative de certains débats sur son sens, continue de constituer une «matière chaude» en vue des de la définition du futur du régime climatique lors de prochaines échéances des négociations à Paris en 2015 et à l'aune de l'état actuel de la crise environnementale planétaire.

Durée dans le temps d'une négociation mondiale axée sur l'atténuation et montée de l'adaptation dans un espace politico-thématique limité : ce sont là deux facteurs de complexité qui rendent ce processus, lourd en enjeux, difficile à saisir dans son ensemble. Et ce, non seulement pour la moyenne des publics que s'y intéressent, mais aussi pour les négociateurs eux mêmes. Comment les sciences sociales et politiques, en se servant désormais d'outils et méthodes numériques, peuvent-elles contribuer à maîtriser cette complexité ? De quelle façon ces outils et méthodes peuvent-ils aider à comprendre la trajectoire, à peine étudiée, de l'adaptation au sein de cette arène ${ }^{6}$ et les enjeux qu'elle soulève à présent ? C'est là le défi auquel s'est attaqué le projet « Cartographier les débats sur l'adaptation» (MEDEA, pour Mapping debates on adaptation) ${ }^{7}$. Et ce, en

${ }^{6}$ Comme l'ont constaté récemement, dans une première tentative de systématisation Khan et Roberts (2013)

7 Pour une description du projet voir la page web: http://www.medialab.sciences-po.fr/fr/projets/medea-mapping-environmentaldebates-on-adaptation/ 
engageant une expérience innovante à l'aide des méthodes et interfaces numériques, avec la production, la circulation et l'éventuelle l'utilisation d'un nouveau type de visualisations du processus de négociation au sein de la CCNUCC. Il s'agira dans cet article de présenter et d'ouvrir à la discussion l'un des résultats de cette expérience. Plus précisément, des graphes produits à partir d'un source de données jusqu'alors inexploitée à cette fin $^{8}$ : les Bulletins de négociations de la terre (BTN, ou ENB pour Earth negotiations bulletin), édités et publiés tous les jours de négociation par l'Institut international du développement durable (IISD pour International Institute for Sustainable Development). Plus précisément, nous avons produit sur cette base: d'une part le réseau sémantique dessinant l'espace thématique des négociations considérées dans leur ensemble, d'autre part un graphe de flux montrant l'évolution de l'importance des thèmes au cours des négociations jusqu'en 2013. Le premier étant essentiellement au service du second, c'est sur ce dernier que nous nous concentrerons $\mathrm{ici}^{10}$.

Nous tâcherons de répondre aux questions suivantes pour présenter notre enquête et ses résultats. Primo, par quelles méthodes et protocoles cette traduction graphique des négociations, que nous dénommons «cartographique» (Venturini, 2010, 2012) ${ }^{11}$, a-t-elle été menée afin d'assurer un changement d'échelle et d'augmenter la lisibilité de l'évolution de l'agenda des COP au cours des vingt dernières années ? Et avec quels résultats ? Secundo, comment une lecture de ces visualisations de données permet-elle d'interroger la dynamique de la controverse entre atténuation et adaptation, les deux composantes du partage le plus fondamental de la politique climatique, au sein de la CCNUCC ? Et, tertio, - en guise de conclusion - quelles perspectives s'ouvrent à partir de l'intégration de ces «cartographies» des négociations pour la recherche scientifique ou l'action publique, notamment dans le cadre des prochaines échéances des négociations?

\section{Changer d'échelle : du terrain des négociations à l'abstraction du}

\section{graphe}

Des négociations à leur représentation sous forme de graphes, le chemin est, en termes latouriens, celui de toute opération de traduction en deux sens qui permet un changement d'échelle tout en assurant la consistance des résultats (Latour, 1993, 1987). Dans le cadre de notre enquête, ce chemin s'est fait par l'application expérimentale des méthodes numériques à un corpus de négociations climatiques. Or, c'est la constitution d'un tel corpus adapté au traitement numérique qui pose, dès le départ, problème. $\mathrm{A}$ ceci s'ajoute ensuite la mise au point d'un protocole d'extraction et d'analyse automatique et manuelle des contenus du corpus afin de produire des données permettant in fine d'aboutir à une modélisation réaliste et robuste mais aussi à une représentation lisible.

\subsection{Constituer un corpus : le BTN comme proxy des négociations}

\footnotetext{
${ }^{8}$ Pour un bilan des savoirs méthodologiques et théoriques en langue française voir (Barats, 2013)

${ }^{9}$ Accessible à l'adresse: http://www.iisd.ca/

${ }^{10}$ Le premier graphe a été abordé en détail par ailleurs (Venturini, Baya-Laffite, Cointet, Gray, Zabban et Pryck, 2014)

${ }^{11}$ Sur l'utilisation et les limites de la métaphore cartographique pour l'analyse du web voir (Plantin, 2013)
} 
Avant toute opération de cartographie, le premier défi est de produire un corpus bien délimité et cohérent à l'aune des questions de recherche. Pour comprendre la spécificité des débats sur l'adaptation planifiée dans l'arène CCNUCC et trouver des stratégies de visualisation pertinentes nous avons travaillé lors d'un certain nombre d'ateliers avec des experts sur les négociations, y compris des négociateurs eux mêmes ${ }^{12}$. Dans ce cadre, l'objectif principal de l'expérience d'inscription visuelle a été conçue pour pouvoir saisir la trajectoire de l'adaptation, en tant que sujet initialement marginal, mais porté depuis le début par les pays les plus vulnérables comme question devant être reconnue en tant qu'élément clé du régime climatique de la CCNUCC. S'agissant alors de déterminer d'abord quantitativement, puis visuellement, le poids absolu et la position relative des questions d'adaptation par rapport à celles relevant de l'atténuation au cours des négociations, force est de reconstruire à partir de données numériques la totalité du processus. Encore, pour visualiser l'évolution dans le temps, les données doivent contenir des marqueurs temporels.

Avant même le travail cartographique, quelles sources choisir pour constituer un corpus qui puisse fonctionner comme un proxy du terrain des COP ? Nous avons abouti à une solution en quelque sorte hybride: les bulletins des négociations de la terre publiés par l'IISD, source la fois experte, informative, et basée sur le terrain des négociations. Ce choix résulte d'un compromis entre contraintes de temps, de ressources, de moyens techniques d'une part, et représentativité, fiabilité, et potentiel pour le travail numérique des documents d'autre part. Précisons donc les trois caractéristiques de la source BTN qui nous ont conduit à la retenir.

Primo, les BTN couvrent l'ensemble du processus officiel des négociations climatiques. Initiative de trois experts participant au Sommet de Rio de 1992, le BTN est depuis devenu un service d'information publié par l'IISD. Actuellement, il fournit des rapports sur 33 processus de négociation onusiens sur l'environnement et le développement. Le Volume 12 est consacré aux négociations sur le climat et comprend les sessions de la $\mathrm{COP}$ ainsi que toutes les réunions liées à la Convention, dont celles des organes subsidiaires et des ateliers thématiques. Chaque jour de négociation, un bulletin est distribué aux participants et mis en ligne sur la page de l'IISD. Le but principal est d'informer quotidiennement les acteurs des négociations des avancées et blocages sur diverses thématiques. L'archive en ligne des bulletins constitue, pour l'ensemble des acteurs - y compris la recherche -, une source unique d'information sur les négociations internationalement reconnue.

Secundo, si ces documents sont fiables, c'est parce que leur écriture est très contrainte. Les bulletins résultent d'un travail collectif ${ }^{13}$. L'équipe du service de rapports de l'IISD est composée, entre autres, de quatre responsables permanents et une soixantaine de consultants experts de trente-deux pays (essentiellement des doctorants ou docteurs, ayant de l'expérience dans les relations entre environnement et développement). Assurer l'uniformité de style ainsi que les traces de subjectivité des rapporteurs multiples est un enjeu capital s'agissant d'un service

${ }^{12}$ Dans le cadre de ces ateliers, nous avons travaillé en étroite collaboration avec François Gemenne et Alexandre Magnan de l'Institut développement durable et relation internationales (IDDRI), avec Amy Dahan et Helène Guillemot du Centre Alexandre Koyré (projet ANR ClimaConf) et avec les partenaires du projet Européen EMAPS (Electronic Maps to Assist Public Science), coordonnée par le medialab Sciences Po.

${ }^{13}$ Voir la page de l'équipe du BTN à l'adresse : http://www.iisd.ca/about/team/ 
d'information, très limité dans sa vocation analytique. Ainsi, chaque numéro passe par des relectures et, en amont, les auteurs et les éditeurs du BTN suivent des formations spécifiques à l'écriture des bulletins. Le «manuel de style» du BTN compile les normes et modèles (templates) visant à éliminer toute forme de subjectivité des auteurs dans les rapports.

Et tertio, si ces documents sont précieux pour un traitement automatique et l'obtention de données d'un processus aussi long et complexe, c'est non seulement parce qu'ils sont fiables, mais aussi parce qu'ils sont extrêmement standardisés : non seulement le contenu des rapports quotidiens est équilibré et étayé par le témoignage de plusieurs participants, mais aussi il est toujours présenté suivant la même structure tant du point de vue de sa mise en forme que de la façon dont les contenus sont restitués. D'une longueur maximale de 1800-1900 mots, ils incluent une introduction et un bref historique de la négociation, puis le déroulé des actes officiels des négociations par thème ou session de chaque jour, et enfin des résultats de la négociation. Ne rentrant pas dans les détails des discussions, ni les nuances des positions, ils proposent en revanche une vue d'ensemble et ce, par une paraphrase, point par point, des arguments échangés entre les participants. L'extrait suivant issue du numéro 588 sur la COP19 tenue en 2013 à Varsovie, et portant sur le volet adaptation dans les négociations relatives à la Plateforme de Durban l'illustre bien :

\begin{abstract}
"Adaptation: ADP Co-Chair Kumarsingh invited parties to examine: how the current adaptation framework could be strengthened; and adaptation in the 2015 agreement, including a proposed global goal.

Many countries recognized the central role of NAPs and underlined the global, regional, national and local dimensions of adaptation. PERU reiterated the proposal by AILAC to use national communications to reinforce NAPs and identify gaps. SAUDI ARABIA said NAPs should be a requirement for all countries. INDONESIA, CHINA and the REPUBLIC OF KOREA stressed the link between adaptation and sustainable development." ${ }^{14}$
\end{abstract}

Si l'on perd en finesse par rapport au matériau orignal (ce qui risque de frustrer l'anthropologue, l'historien ou l'analyste du discours politique par exemple), la ressource BTN reste cependant optimale à nos fins : des comptes-rendus marqués temporellement (qui plus est précisant les modalités d'énonciation ${ }^{15}$ ) constitués en corpus numérique englobant de façon exhaustive l'ensemble du processus de négociation. Puisque c'est l'équipe du BTN qui a, pour ainsi dire, fait le travail traditionnel de « terrain », le travail de traduction, tel que nous l'avons mené, se fonde sur une "délégation» spécifique à l'économie des recherches axées sur le traitement textuel automatique. Le BTN fonctionne ainsi comme un enquêteur de terrain par procuration: c'est là que l'on retrouve le sens étymologique du terme anglo-saxon "proxy», que nous utilisons pour caractériser notre corpus. Or, que le travail de terrain soit délégué n'implique pas l'abandon des méthodes «non-numériques ». Loin de là, toute l'expérience de cartographie numérique, telle que nous l'avons conçue, implique un recours constant aux méthodes traditionnelles de la recherche sociologique, par des entretiens et ateliers avec les acteurs du

\footnotetext{
${ }^{14}$ (IISD, 2013b)

${ }^{15}$ Ceci est une condition pour l'éboration d'autre type de visualisations, en l'occurrence des liens entre acteurs et questions de débat, que nous avons aussi éleborées et que nous présentons sommairement ailleurs (Venturini, BayaLaffite, Cointet, Gray, Zabban et De Pryck, 2014)
} 
terrain et les experts du domaine et une revue manuelle de la documentation primaire et la littérature secondaire : pour formuler des questions, pour construire les corpus, pour en extraire les données, pour les visualiser, pour les analyser, pour les mettre en valeur, on ne peut pas s'en passer. C'est là une précision certes quelque peu banale, mais qu'il convient de rappeler, notamment au vu des discours ambiants, enthousiastes ou défaitistes, quant aux méthodes numériques.

\subsection{La main et la machine : la création des données pour visualiser le}

\section{déploiement topologique et temporel des thèmes des négociations}

Si les BTN présentent des avantages comme source pour suivre le processus de négociation, il faut encore surmonter les difficultés posées par le traitement du contenu afin de pouvoir structurer les données, calculer les cooccurrences qui sous-tendent les ensembles thématiques relevant de l'atténuation et de l'adaptation, et enfin les visualiser. C'est là le deuxième défi de la traduction des négociations en graphe, défi que requiert une connaissance fine du corpus étudié, des méthodes d'analyse de contenu et de réseaux complexes et surtout du domaine sur lequel porte l'enquête, en l'espèce les négociations climatiques.

Notre corpus a été constitué, à partir de la page web contenant les 594 éditions du vol. 12 du BTN ${ }^{16}$. Il se compose de la totalité des daily issues, depuis la dernière session du Comité international de négociation de la Convention (INC) juste avant la première COP à Berlin, en 1995, jusqu'à la COP de Varsovie en 2013 (COP19). Pour assurer sa cohérence, nous avons écarté les éditions relatives aux sessions autres que les COP, notamment celles de l'Organe subsidiaire de conseil scientifique et technologique (SBSTA, Subsidiary Body for Scientific and Technological Advice) et de l'Organe Subsidiaire pour la mise en application (SBI, Subsidiary Body for Implementation). Pour son exploitation, nous avons utilisé la plate-forme d'analyse de corpus textuels CorText ${ }^{17}$, outil qui se fonde d'une part sur des méthodes de traitement du langage et d'autre part sur des méthodes d'analyse et de visualisation de réseaux complexes. Le protocole d'exploitation du corpus mis en place se décline en trois étapes itératives, mettant en jeu, d'une part, les algorithmes opérés par une machine et, d'autre part, le paramétrage de ces derniers ou certaines opérations purement manuelles de traitement des données.

La première étape consiste à extraire du contenu textuel les groupes nominaux définissant les objets de négociation. Pour le traitement du contenu textuel, nous avons extrait des bulletins 5663 paragraphes, chaque paragraphe des Bulletins correspondant à une unité thématique naturelle selon les normes de production et de présentation du BTN. Un analyseur morphosyntaxique a permis d'identifier l'ensemble des groupes nominaux présents. Ces derniers sont regroupés au sein de classes sémantiques homogènes regroupant les formes équivalentes. Seules les classes - que nous appellerons par la suite « termes » par simplicité - les plus fréquentes (au moins 7 occurrences) ont été conservées. La deuxième étape consiste en un tri manuel des expressions les plus pertinentes figurant dans cette liste, afin de ne conserver que des termes significatifs. C'est-à-dire, des

\footnotetext{
${ }^{16}$ Accessible à l'adresse: http://www.iisd.ca/vol12/

${ }^{17} \mathrm{La}$ platerforme CorText est l'outil en ligne d'analyse de corpus textuels développé par l'équipe CorText (http://www.cortext.org) de l'IFRIS (Institut Francilien Recherche Innovation Société), elle est disponible à l'adresse : http://manager.cortext.net/
} 
termes qui se réfèrent à des questions identifiables (par exemple « responsabilité historique »). Il s'agit donc de rejeter tous ceux qui ne sont pas assez précis (par exemple "groupes de contact», "des efforts redoublés ») ou qui sont trop structurants (par exemple les noms des organes subsidiaires de la CCNUCC). D'autre part, il est nécessaire de vérifier manuellement la fusion des formes équivalentes des termes, des synonymes et acronymes. Dans une troisième étape, un réseau sémantique est construit à partir de la matrice de cooccurrence de l'ensemble des termes pertinents. Chaque élément de cette matrice associe à un couple de termes la fréquence des événements de co-apparition au sein d'un même paragraphe. La force des liens qui relient les nœuds de ce réseau sémantique résulte du calcul d'une mesure de proximité ${ }^{18}$ appliquée à la matrice de cooccurrence. Un algorithme de détection des communautés (Blondel, Guillaume, Lambiotte et Lefebvre, 2008) est ensuite utilisé pour identifier les sous-groupes cohésifs du réseau dont nous faisons l'hypothèse qu'ils regroupent le vocabulaire spécifique aux différents thèmes-cadres de la négociation. Une représentation visuelle sous forme de carte mettant en exergue cette catégorisation est enfin produite.

Pour parvenir à des résultats visuels robustes, un grand nombre d'itérations ont été nécessaires entre les textes du corpus, les données et les statistiques issues des extractions automatiques, et des prototypes de visualisations du réseau sémantique. Aucune visualisation des termes extraits ne pouvait être «lue» sans ces interventions itératives. D'une liste de 1178 termes, nous avons limité le dictionnaire des termes à environ 300 groupes nominaux grâce à un nettoyage manuel des données qui a permis de réduire le « bruit » et d'atteindre un degré satisfaisant de complexité, tout en assurant la lisibilité des graphes ${ }^{19}$. Comment savons-nous que ce qu'on éliminait était du «bruit» et pas un «signal»? Ces interventions itératives ne se font pas dans l'ignorance du thème étudié. D'une part, elles s'appuient sur les connaissances des négociations que les chercheurs impliqués ont par ailleurs; d'autre part, sur celles qui doivent être recherchés à partir de sources primaires et secondaires (entretiens, ateliers, revue de la littérature).

Ainsi, si nous avons pu échapper à la fameuse régression de l'expérimentateur signalé par Harry Collins dans son étude sur la détection des ondes gravitationnelles supposées par la théorie grâce à des appareillages mis au point ad hoc (Collins, 1992), c'est parce que, à la différence des chercheurs étudiés par Collins, nous avions une vision $a$ priori de certains éléments attendus de cet exercice cartographique. Ainsi, si l'on peut dire que les méthodes et protocoles cartographiques mis en place sont fiables sur notre corpus, c'est parce les résultats sont compatibles avec les connaissances établies par ailleurs sur le «territoire » cartographié (par exemple par rapport au corpus étudié si l'on lissait «manuellement», et in fine par rapport aux négociations elles mêmes). Sans cette connaissance, on ne saurait dire si le territoire a été fidèlement cartographié au vu de l'application expérimentale des méthodes. Méthodes numériques et méthodes traditionnelles loin de se substituer, se combinent et se complémentent afin de produire des résultats substantiels spécifiques. Dans quelle mesure ce ne sont pas ces connaissances (ou hypothèses) préalables qui ont conduit les chercheurs impliqués, compte tenu du

\footnotetext{
${ }^{18}$ Précisément, nous avons employé l'équation 18 (difference-weighted MIbased model) de la référence: (Weeds et Weir, 2005)

${ }^{19}$ Les deux listes sont disponibles à l'adresse: http://medialab.sciencespo.fr/publications /malentendus /)
} 
caractère novateur des méthodes utilisées, à façonner la carte jusqu'à obtenir un résultat lisible conforme à ce qu'on savait (ou supposait) par ailleurs? C'est là une question délicate que certaines de ces nouvelles méthodologies peuvent parfois susciter. On peut cependant répondre ainsi : les algorithmes adossés à la robustesse des processus empiriques présents dans les données peuvent au contraire agir comme garde-fou pour infirmer des hypothèses infondées.

Le résultat de l'analyse de réseau sémantique entre termes extraits est l'identification de 12 grands thèmes qui structurent les négociations. Après spatialisation du réseau par le logiciel Gephi ${ }^{20}$, nous aboutissons à la carte finale (fig. 1). Le réseau regroupe ainsi 300 nœuds qui sont autant d'expressions nominales qui animent les négociations climatiques entre 1995 et 2013. Les intitulés des clusters ont été attribués manuellement selon les grands thèmes qu'ils mettent en évidence.

Pour explorer la robustesse et les possibilités d'exploitation des données et leur visualisation, nous avons organisé des ateliers dans une approche expérimentale de coproduction de dataviz avec ses utilisateurs potentiels, comprenant entre autres des chercheurs et des négociateurs ${ }^{21}$. Empruntant à l'approche des méthodes numériques de la Digital Methods Initiative de l'Université d'Amsterdam, elle consiste en réunir pendant cinq jours quelques 30-40 personnes - chercheurs, programmeurs, designers et experts - pour travailler ensemble sur d'exploitation de jeux de données afin de créer des visualisations sur les éléments de controverses identifiés par les experts invités durant la première journée. Tenu à Sciences Po Paris du 6 au 10 Janvier 2014, le "data sprint» sur les négociations de l'adaptation a réuni 37 participants ${ }^{22}$. Ces derniers ont été ensuite répartis en groupes de 4-6 participants pour s'attaquer chacun à un challenge au cours des 4 jours suivants (Yamin, 2014).

La visualisation diachronique (fig. 2) a été mise au point dans le cadre de cet atelier pour répondre à l'un des défis soulevés par Farhana Yamin, négociatrice pour l'Alliance des petits États insulaires (AOSIS), à savoir dans quelle mesure l'atténuation et l'adaptation occupent, à différents moments, l'espace des COP. Elle a été produite à partir de la catégorisation thématique fournie par CorText manager sur l'ensemble de la période pour attribuer en retour à chaque paragraphe du corpus BTN le ou les thèmes qu'il aborde. Le contenu des paragraphes est comparé aux distributions des termes dans chaque cluster. Un paragraphe qui partage un nombre significatif de termes avec un cluster sera associé à ce dernier. Le décompte du nombre de paragraphes par thématique à chaque COP nous a permis de construire cette seconde visualisation des négociations en utilisant le logiciel Raw, produit par Density Design (Politecnico di Milano). Cette fois, la visualisation met en valeur les flux diachronique de l'ensemble des douze thèmes structurant l'espace thématique des négociations, et représentant leur présence relative et absolue d'une COP à l'autre entre 1995 et 2013. Le volume absolu de chaque catégorie est

${ }^{20}$ La taille de nœuds est proportionnelle à leur fréquence dans l'ensemble du corpus. La position des nœuds est déterminée par l'algorithme de force vectorielle, Force Atlas 2, conçu pour le logiciel Gephi (Jacomy, Venturini, Heymann et Bastian, 2014)

${ }^{21}$ Sur la méthode des Sprints voir: (Venturini, Ricci, Mauri, Kimbell, et al., 2015)

${ }^{22}$ Le groupe d'experts invités comprenait Richard Klein (Stockholm Environment Institute), Frédéric Glanois (Ministère français des Finances), Farhana Yamin (Chatham House), Kees van der Geest

(Université des Nations Unies) et Alix Mazounie (Réseau Action Climat) 
représenté par l'épaisseur du flux et est susceptible de varier à chaque COP. La présence relative d'un thème une année donnée est définie par son rang (ordonné de haut en bas sur le diagramme) ${ }^{23}$.

L'expérience d'exploitation du corpus BTN que nous avons réalisée témoigne donc d'un processus de production d'analyses et de synthèses nouvelles aboutissant à « des cartes » comme produit final. Cet artefact ouvre la possibilité de narrations nouvelles dans des agencements incertains où elle pourrait venir à fonctionner comme objet frontière.

\footnotetext{
${ }^{23}$ Pour une version permettant de mettre en avant un thème parmi les douze s'adresser à la version en ligne disponible à http://climaps.org/\#!/map/absoluteand-relative-visibility-of-issues-in-unfccc-negotiations-1995-2013
} 
Figure 1. Graphe du réseau sémantique du BTN configurant l'espace thématique des $C O P^{24}$.

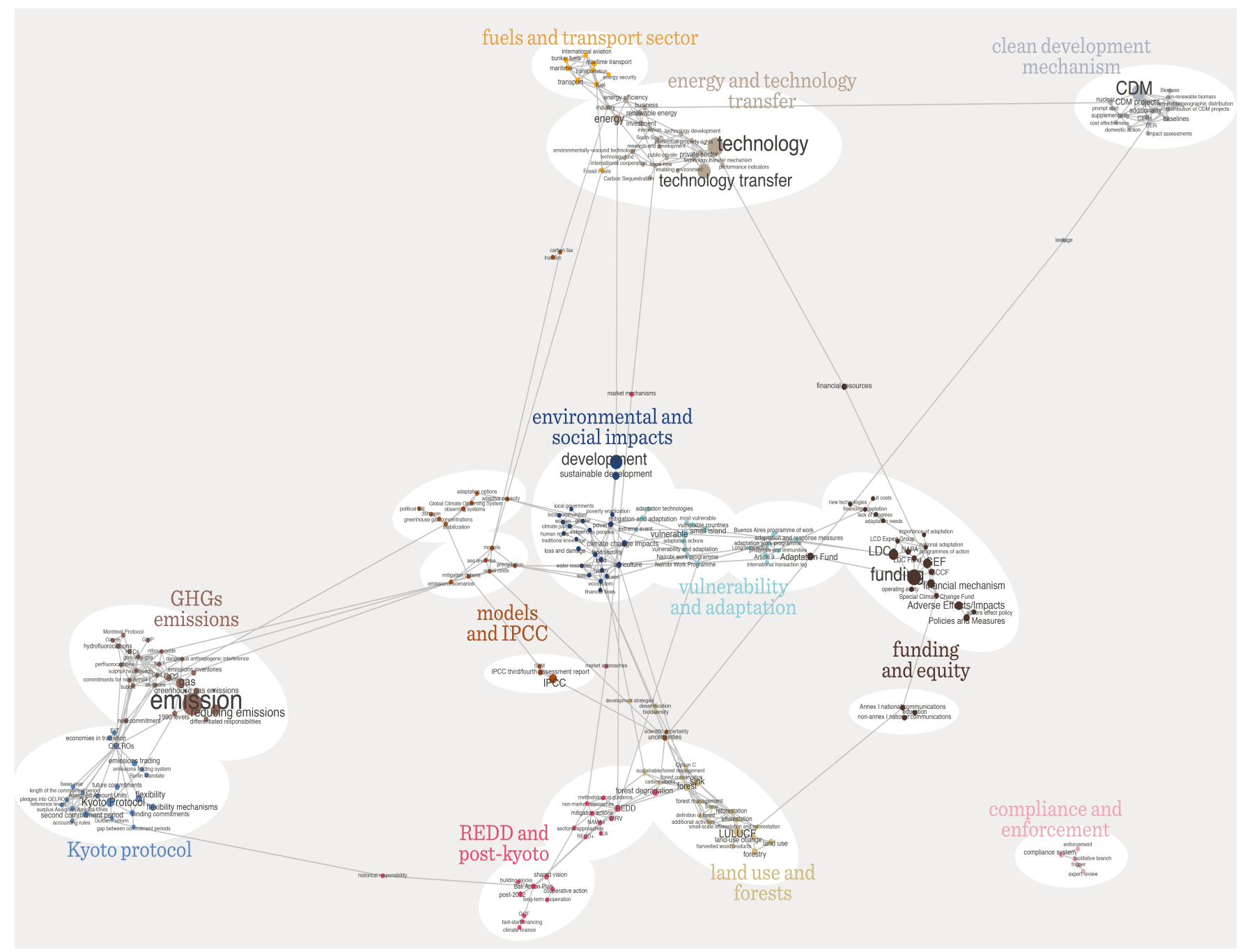
${ }^{24}$ Une version en haute définition de la carte est disponible sur http://medialab.sciences-
po.fr/publications/misunderstandings/figure-1 
Figure 2. Graphe de flux représentant la présence relative et absolue des 12 thèmes cadre des négociations entre 1995-2013

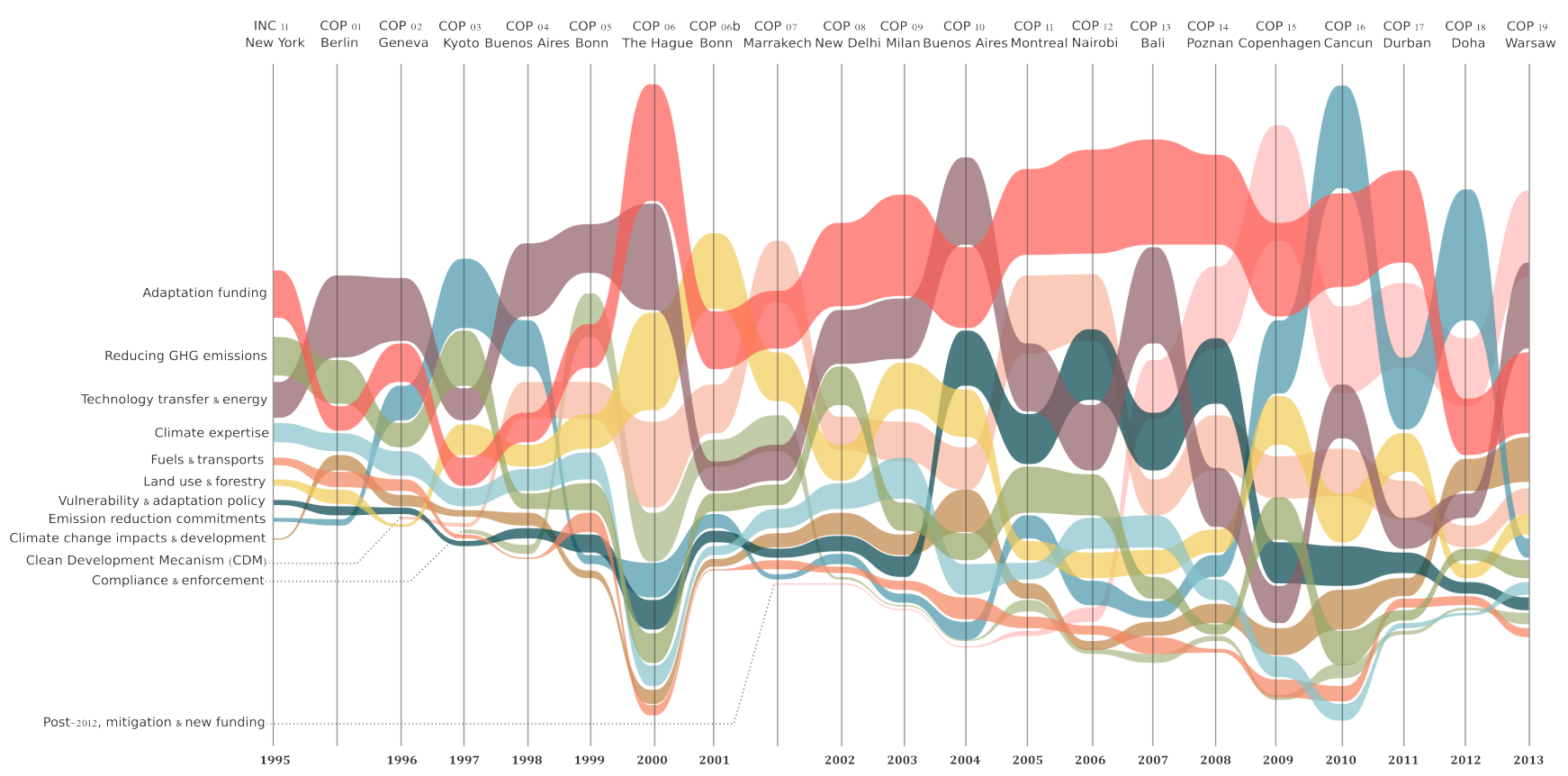




\section{Red(u)ire la complexité : des visualisations des données à la reconstruction}

\section{narrative de la trajectoire de l'adaptation}

Qu'est-ce que ces visualisations des données BTN peuvent nous apprendre sur les négociations? Que le processus de cartographie numérique s'appuie sur ce que l'on peut trouver ailleurs sous une forme textuelle (en commençant par le contenu $\mathrm{du}$ BTN), ne veut pas dire que la visualisation n'apporte rien de nouveau en termes de production de connaissance: elle apporte une nouvelle synthèse synoptique des trajectoires thématiques des négociations. Or, c'est uniquement lorsque les visualisations sont agencées qu'elles intéressent par leur capacité de borner et de donner force à la production de discours. Et ce, grâce à leur capacité d'augmenter la mobilité et l'immutabilité de ce qu'elles représentent avec l'autorité que les méthodes et sources utilisées pour leur production leur confèrent pour, soit infirmer, soit confirmer, des discours sur les négociations. Et si les graphes ne parlent pas, ils ont, cependant, une «affordance»: ils traduisent, représentent, font abstraction, et permettent de maîtriser visuellement la complexité. Entre potentialisation et contrainte interprétative ${ }^{25}$, ils font réfléchir sur ce qu'ils mobilisent; ils font parler d'eux par un effet d'étonnement actif du fait inhabituel, inattendu ou difficile à expliquer des images, ou de certains aspects de leur contenu. D'une part, on peut reconnaître sur le graphe nombre de «moments» (ou «termes» dans le cas de la visualisation synchronique) bien documentés dans la littérature. Mais d'autre part, on peut se surprendre et s'interroger sur nombre d'aspects des graphes (pics des flux, variations, liens entre nœuds, taille des nœuds, classification de certains termes) qui nécessitent une explication supplémentaire. Ainsi, l'un des atouts majeurs de cette mise en image de processus politiques complexes par certains côtés rébarbatifs comme celui des négociations climatiques, consiste à générer un sentiment conscient de perplexité, au sens que les philosophes de la Grèce ancienne accordaient au $\theta \alpha v \mu \alpha ́ \zeta \varepsilon t v$ (émerveillement) ${ }^{26}$. Et partant, ce sentiment déclenche, dans des agencements «carte-utilisateur» divers, une attitude réflexive (politique, épistémique) chez les utilisateurs (y compris les concepteurs) les menant à identifier et analyser où et comment des questions les concernant sont représentées.

Compte tenu de la spécificité sémantique du langage de la CCNUCC (chaque terme composant les thèmes relève d'une histoire propre qui ne se dévoile pas

\footnotetext{
${ }^{25}$ (Hutchby, 2001),

${ }^{26}$ Thaumazein, qui signifie aussi émerveillement, cf (PlATON, 1976, 155D) (Aristote, 2002, I.ii.982b11-24.)
} 
seule) la lecture des visualisations nécessite d'une connaissance fine des négociations. Bien qu'accompagnés de textes plus ou moins explicatifs, ces graphes visent, avant tout, un public déjà informé ou en tout cas «motivé ». Dès lors, plusieurs stratégies narratives sont possibles pour retraduire verbalement la complexité en fonction des agencements dans lesquels le graphe s'inscrit ${ }^{27}$. Aux fins cet article, nous proposons une retraduction du graphe diachronique (figure 2) essentiellement illustrative, nous focalisant sur la question qui guide notre enquête: la place de l'adaptation dans l'espace des négociations. Dans des encadrés sur les images s'affiche le cluster du réseau sémantique correspondant à la thématique tandis que la période d'intérêt est soulignée par un cadre bleu. Dans la mesure du possible, nous reprenons en italiques les termes de chaque cluster thématique de la visualisation synchronique (figure 1), dont l'évolution représentée dans chaque figure est analysée ${ }^{28}$.

Figure 3. Graphe de flux représentant la présence relative et absolue des 12 thèmes cadre des négociations 1995-2013, soulignant deux points d'inflexion en 2000 et 2009

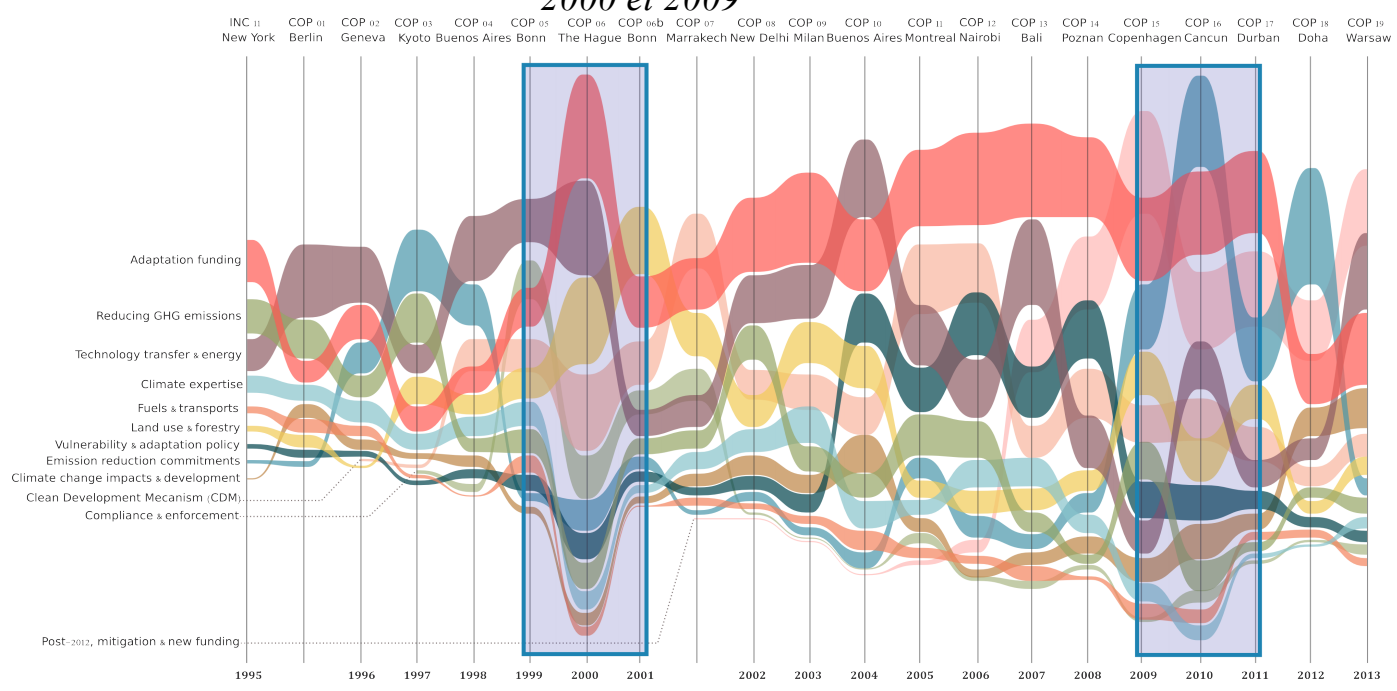

Nous structurons le récit sur la base de deux points d'inflexion identifiés sur la figure 3 et que nous caractériserons dans la suite: celui de la COP6 à La Haye,

\footnotetext{
${ }^{27}$ (Ewick et Silbey, 1995) (Yau, 2013)

${ }^{28}$ Le lecteur peut se référer à la version zoomable du graphe de réseau sémantique en ligne accessible à l'adresse suivante http://www.medialab.sciencespo.fr/publications/misunderstandings/figure-9)
} 
première crise majeure des négociations ; puis celui de la COP15 à Copenhague, second moment clé des négociations. Partant, nous pouvons découper l'histoire de l'adaptation dans les négociations en trois périodes. Ce n'est que depuis la crise des négociations achoppant sur la mise en ouvre du Protocole de Kyoto à La Haye, en 2000, que le thème du financement de l'adaptation, puis celui de l'action d'adaptation, deviennent importants, au moins jusqu'en 2009, lorsque la COP de Copenhague et la définition de nouveaux engagements d'atténuation regagneront le haut de l'agenda.

\section{1. À l'ombre de l'atténuation, l'adaptation portée par les plus vulnérables} (1995-2000)

Si longtemps l'adaptation a été un sujet « tabou » pour d'aucuns, cela n'implique pas qu'elle ait été absente des négociations. Bien au contraire, elle fait l'objet de négociations dès le début.

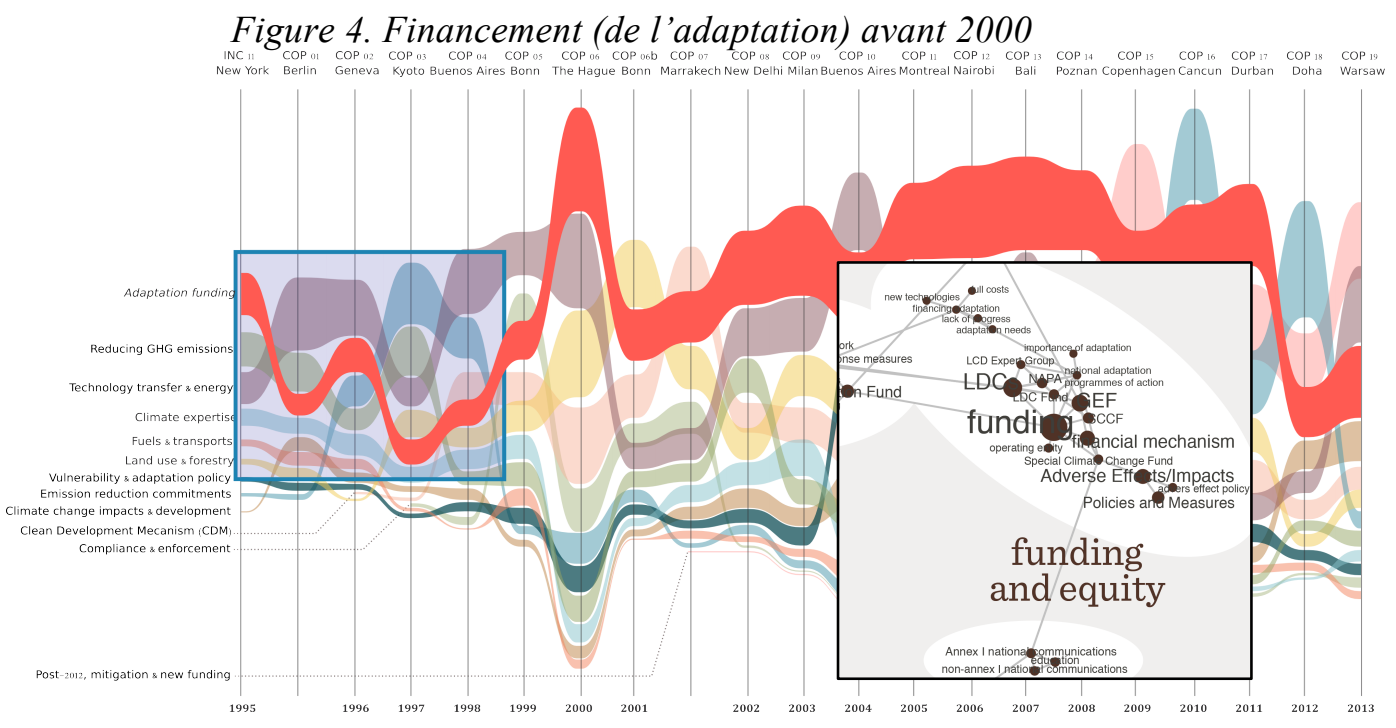

Le financement, notamment de l'adaptation, une priorité ancienne. Les questions du financement de la lutte contre le changement climatique (notamment des pays les moins avancés (PMA), pour lesquels les ressources financières pour faire face à leurs besoins d'adaptation sont les plus critiques) dominent le haut du graphe. Le thème occupe certes une place moins élevée que les questions d'atténuation dans l'agenda, mais il apparaît toujours parmi les principaux thèmes 
lors des premières COP (fig. 4). Sa présence en hausse constante est le résultat de l'action conduite par l'Alliance des petits États insulaires (AOSIS), depuis sa création en 1991, le plus actif des groupements en ce qui concerne l'intégration les questions de vulnérabilité, d'adaptation et de financement de l'adaptation dans la CCNUCC (Betzold, Castro et Weiler, 2012). Par exemple, c'est à sa demande, lors de l'élaboration du texte final de la Convention en 1992, que l'on a ajouté la disposition à l'article 4.4 selon laquelle les pays développés «aident également les pays en développement parties particulièrement vulnérables aux effets néfastes des changements climatiques à faire face au coût de leur adaptation auxdits effets ». C'est là une solution de compromis, l'AOSIS proposant la formulation «à faire face aux coûts de leur adaptation », ce qui pouvait impliquer la totalité des coûts de l'adaptation (Yamin, 2005).

Figure 5. Vulnérabilité et adaptation comme objet politique avant

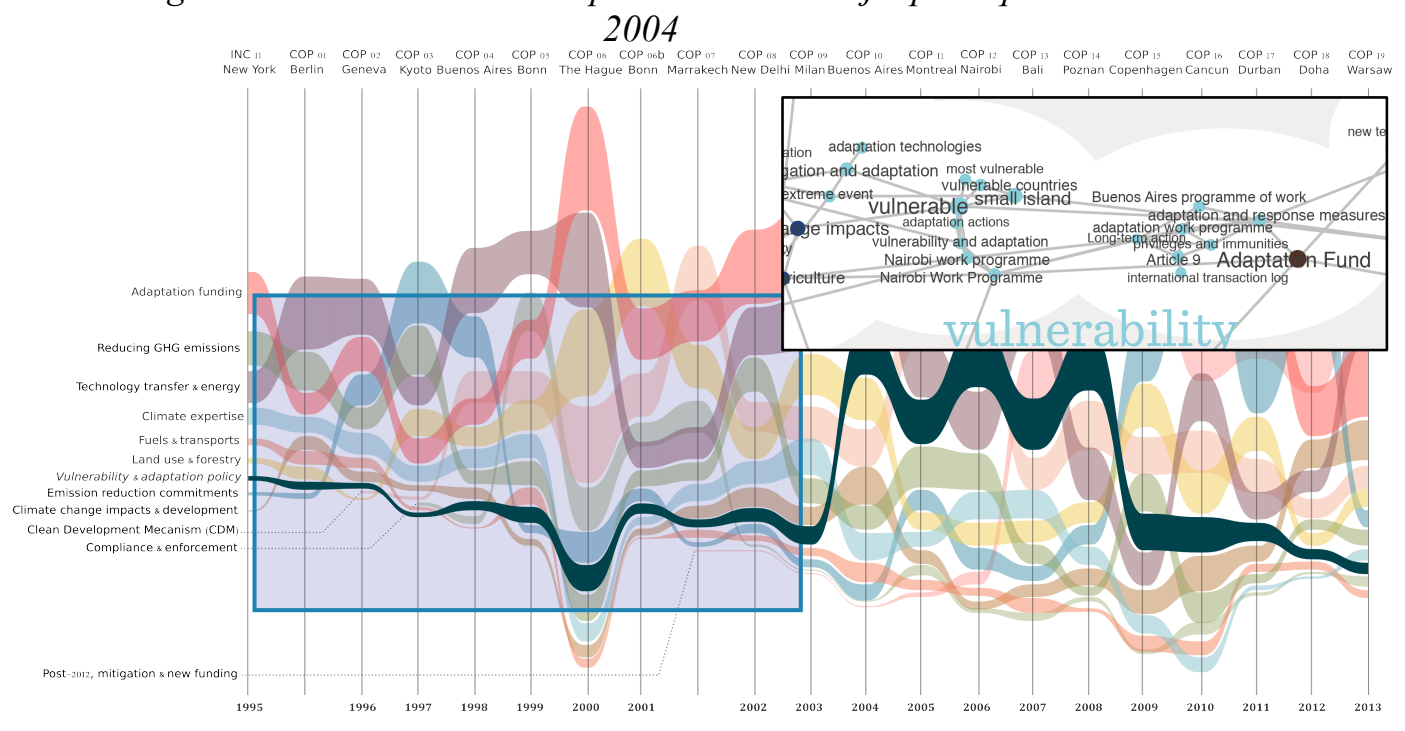

Les programmes d'action en matière d'adaptation des plus vulnérables tardent à s'imposer. Ce qui apparaît comme une question véritablement reléguée au cours des années 1990 (fig. 5) c'est l'action en matière d'adaptation des plus vulnérables au changement climatique et aux évènements extrêmes et autres impacts qui en découlent. Malgré les efforts de plaidoyer de l'AOSIS, la question est mise en attente depuis Berlin. D'une part, c'est là la conséquence de l'approche en trois étapes, donnant la priorité à l'observation, qui est alors décidée. Il s'agit d'identifier quels sont les pays ou les régions particulièrement 
vulnérables aux impacts du changement climatique. Ensuite, la priorité devrait passer à la définition des mesures qui peuvent être prises pour préparer l'adaptation, par exemple quel type de renforcement des capacités est nécessaire en fonction des options d'adaptation à disposition. Ce n'est que dans un troisième temps que l'accent est mis sur la mise en œuvre des mesures permettant de faciliter une adaptation appropriée, par exemple par des technologies d'adaptation. Les raisons de cette approche par étapes viennent des débats que l'adaptation suscite sur le plan conceptuel, normatif et pratique et ce jusqu'à très récemment (Schipper, 2006). D'une part, pour nombre d'acteurs et observateurs craignant que l'adaptation ne devienne une distraction, voire un renoncement à l'objectif fondamental d'atténuation (Godard, 2010), elle est un thème à bannir. D'autres, en revanche, constatent avec inquiétude exactement l'inverse (Pielke, 1998), soulignant comme impératif et juste que l'on réponde sans délai aux conséquences du réchauffement climatique, notamment dans les pays les plus pauvres et vulnérables, indépendamment de tout effort d'atténuation (Huq et Reid, 2004). Ces débats tiennent au caractère ambigu du concept sur le plan normatif, mais aussi sur le plan pratique. Les impacts et la vulnérabilité, notions par essence locales, ne trouvent pas leur place comme objets d'une action collective mondiale en matière d'adaptation, dont les bénéfices sont aussi locales, au sein de la Convention. De plus, puisque l'adaptation est un processus, et non pas un résultat, il est difficile de fixer des objectifs et moyens d'action clairs, donnant lieu à, par exemple, un "protocole d'adaptation ». Dans la mesure où le cadrage de l'adaptation comme objet de politiques n'est pas établi, elle est difficilement institutionnalisable. C'est là une situation qui ne commencera à changer qu'à partir de 2001 avec la publication du troisième rapport du GIEC dans un régime d'atténuation fragilisé par ses échecs. 
Figure 6. Emissions de GES

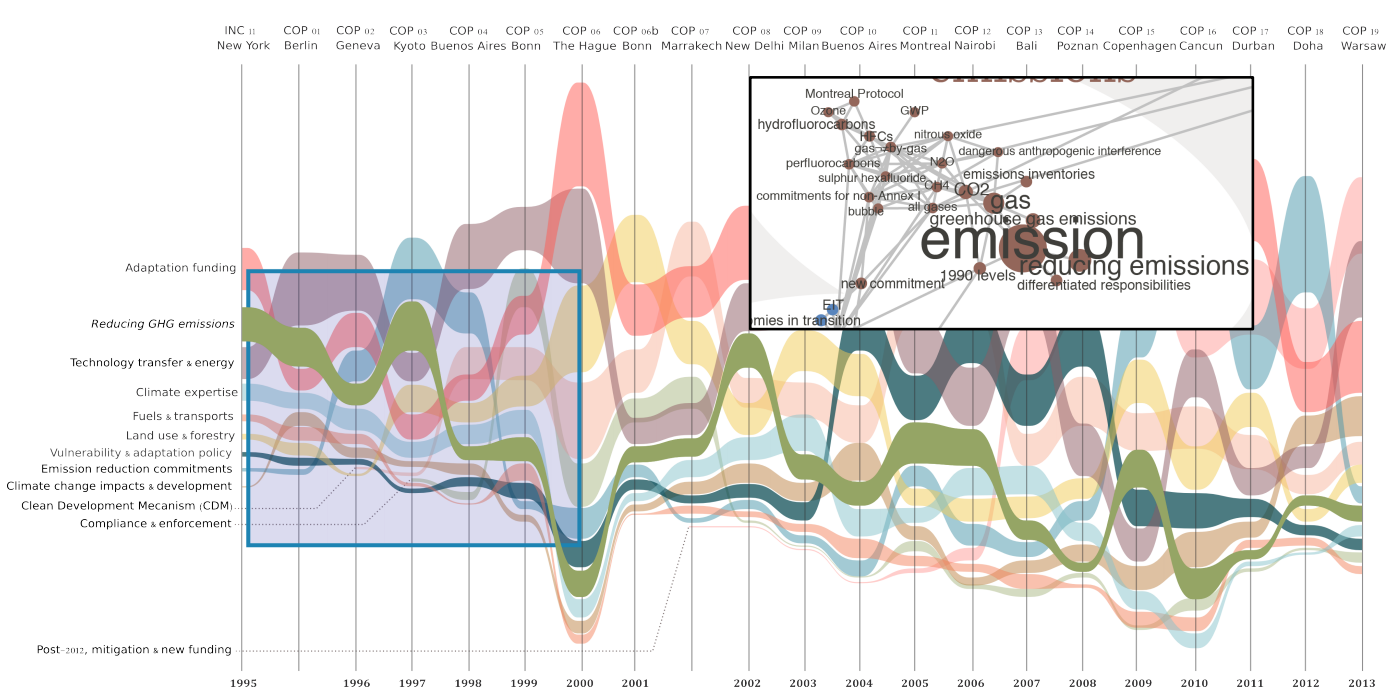

Figure 7. Engagements de réduction des GES (Protocole de Kyoto et post Protocole Kyoto)

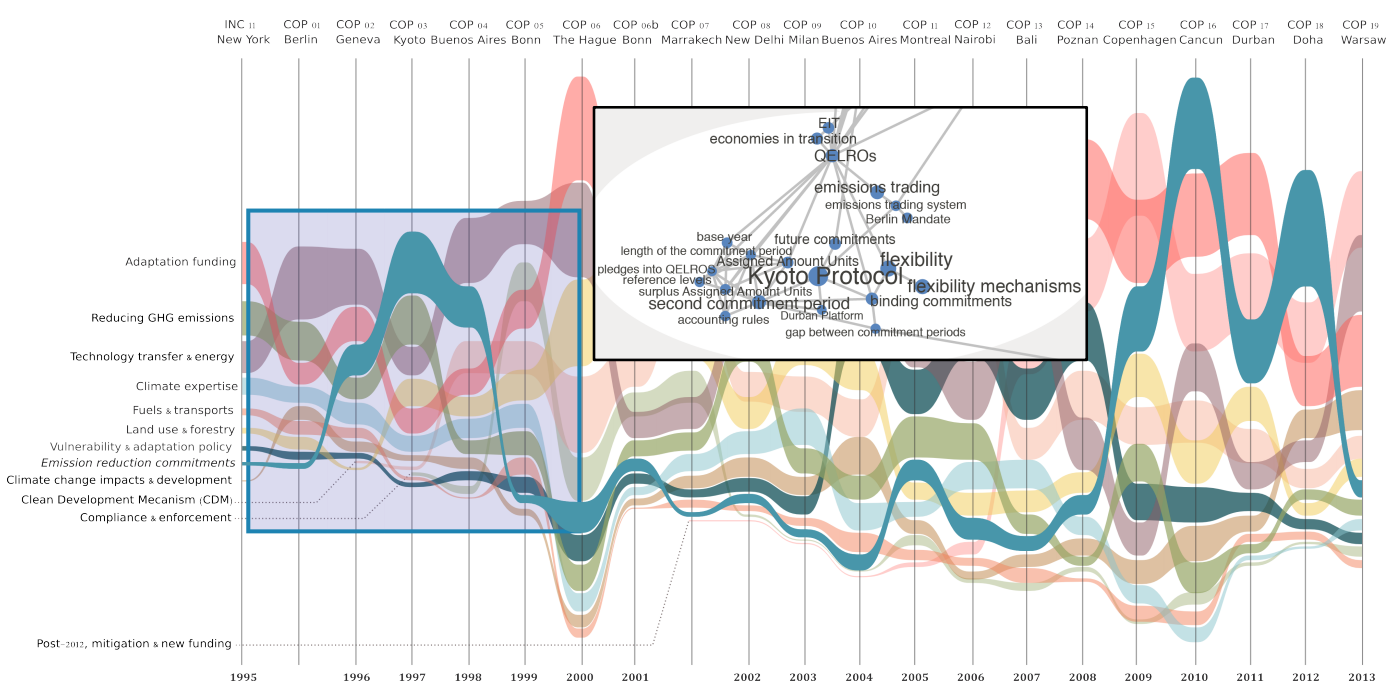


Engagements de réduction des émissions de GES. De quoi discute-t-on alors pendant les six premières COP ? On le voit bien : depuis le Mandat de Berlin, l'accent est mis sur l'objectif de réductions des émissions de GES pour éviter une perturbation anthropique dangereuse du système climatique. Le sujet atteint son premier pic à l'agenda à la COP3, dont est issu le Protocole de Kyoto (PK). Le PK établit des engagements contraignants et quantifiés de limitation et de réduction des émissions (QELROS) de six GES pour les 41 pays industrialisés et les pays en transition vers une économie de marché. A l'aune du principe de responsabilités communes mais différenciées, le but est d'atteindre une réduction globale d'au moins $5,2 \%$ en dessous des niveaux de 1990 et ce sur une période d'engagement s'étendant de 2008 à 2012. Pour atteindre ce but, le PK prévoit des mécanismes de flexibilité, dont le marché d'émissions et le mécanisme de développement propre, permettant aux pays ayant une obligation de réduction de la réaliser à moindre coût dans un autre pays.

Figure 8. Énergie et transfert de technologies

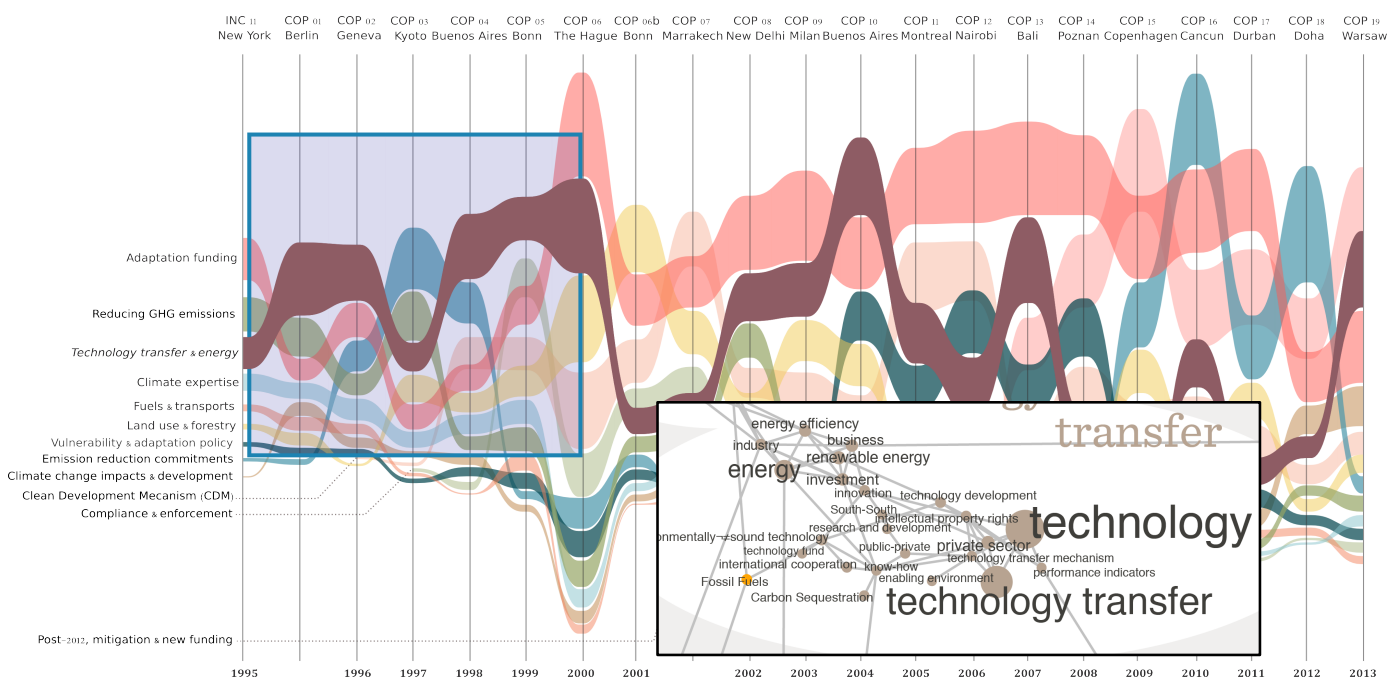

Énergie et transfert de technologies. A l'aune des objectifs d'atténuation en fonction des responsabilités différenciées, les questions de transferts de technologie des pays industrialisés vers les PED, notamment en matière d'énergie, sont centrales, comme le montre la trajectoire du thème au cours des premières COP, jusqu'à La Haye, mais aussi par la suite (fig. 8). La négociation à ce sujet portera, entre autres, sur les mesures tendant à créer un environnement 
favorable à l'investissement des entreprises du secteur privé (qui joue un rôle central dans l'innovation et le développement des technologies) d'une part, et l'établissement de mécanismes de coopération et de financement, permettant aux PED d'engager une transition vers des énergies robustes du point de vue de l'environnement, renouvelables et efficientes.

Figure 9. Puits de carbone (forêts) et règles LULUCF 1997-2004

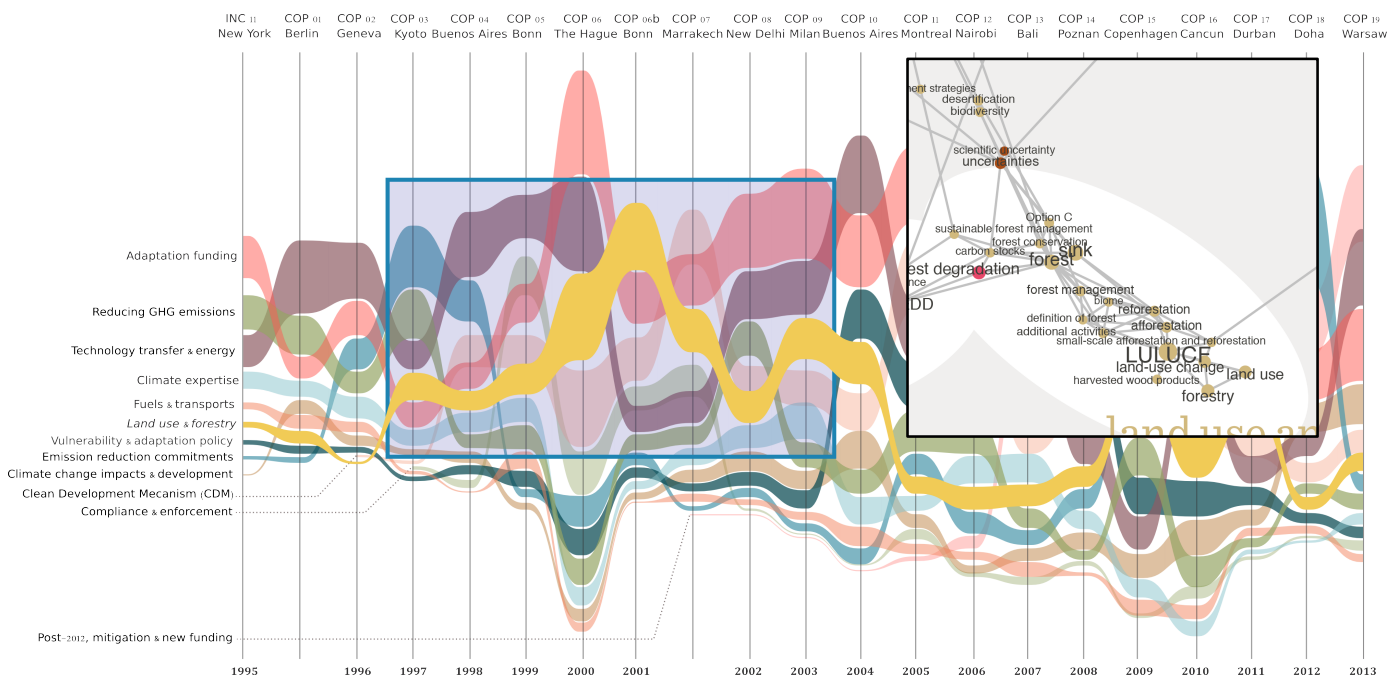

Comptabilité des puits de carbone. La définition des règles de comptabilité des réductions fait l'objet des négociations durant les quatre années qui suivent l'adoption du Protocole de Kyoto $(\mathrm{PK})$, conduisant à la crise de La Haye (Dessai, Lacasta et Vincent, 2003). C'est alors, comme nous pouvons le voir figure 9, que la prise en compte des puits de carbone, dont les forêts, entre progressivement en scène. La montée (tant en termes absolus que relatifs) que l'on peut observer tient aux controverses qui l'entourent. Alors que certains pays allèguent que le stockage de carbone, permettant d'atteindre les objectifs de réduction, doit être compris dans le PK, d'autres prétendent l'exclure craignant que le changement d'affectation des terres et de la foresterie (LULUCF, pour Land use and land use change and forestation) ne devienne une excuse pour contourner des obligations de réduction des émissions liées aux énergies fossiles. La controverse atteint son paroxysme à la COP6 de La Haye, et- suite à l'impasse dans laquelle stagne la négociation- à la COP6bis, tenue à Bonn en juin 2001. L'urgence de trancher se 
voit encore renforcée depuis l'annonce en mars 2001 du refus des Etats-Unis de ratifier le $\mathrm{PK}$, ces règles de calcul étant la clé de voûte pour assurer le ralliement de certains pays au protocole et éviter qu'il soit entièrement vidé de son sens. La définition des activités couvertes par le secteur LULUCF et des règles de comptabilisation de ces activités a été résolue avec l'établissement des Accords de Marrakech lors de la COP7, fin 2001. Or, le travail ne sera achevé qu'à l'issue de la COP10, à Buenos Aires.

\subsection{La traversée du haut plateau de l'adaptation (2000-2009)}

Début années 2000, on assiste à un point d'inflexion. Nous le voyons bien sur les figures suivantes : la question de l'adaptation des plus vulnérables gagne sa place de COP en COP : en tant qu'objet de financement depuis La Haye (2000) (fig. 10), et en tant qu'objet de mesures politiques depuis Buenos Aires (2004) (fig. 5). C'est-ce que l'on peut appeler, « la traversée du haut plateau de l'adaptation » du fait de la forme prise par la trajectoire de ces deux sujets sur le graphe ${ }^{29}$.

\footnotetext{
${ }^{29}$ Ailleurs on a parlé du « hockey stick » pour le controversé graphe de Michel Mann, demontrant la hausse de la température anormale depuis les dernières décennies grâce d'une reconstruction par des données proxy de la température au cours de mil ans (puis élargi à deux mille ans). (Mann, 2013)
} 


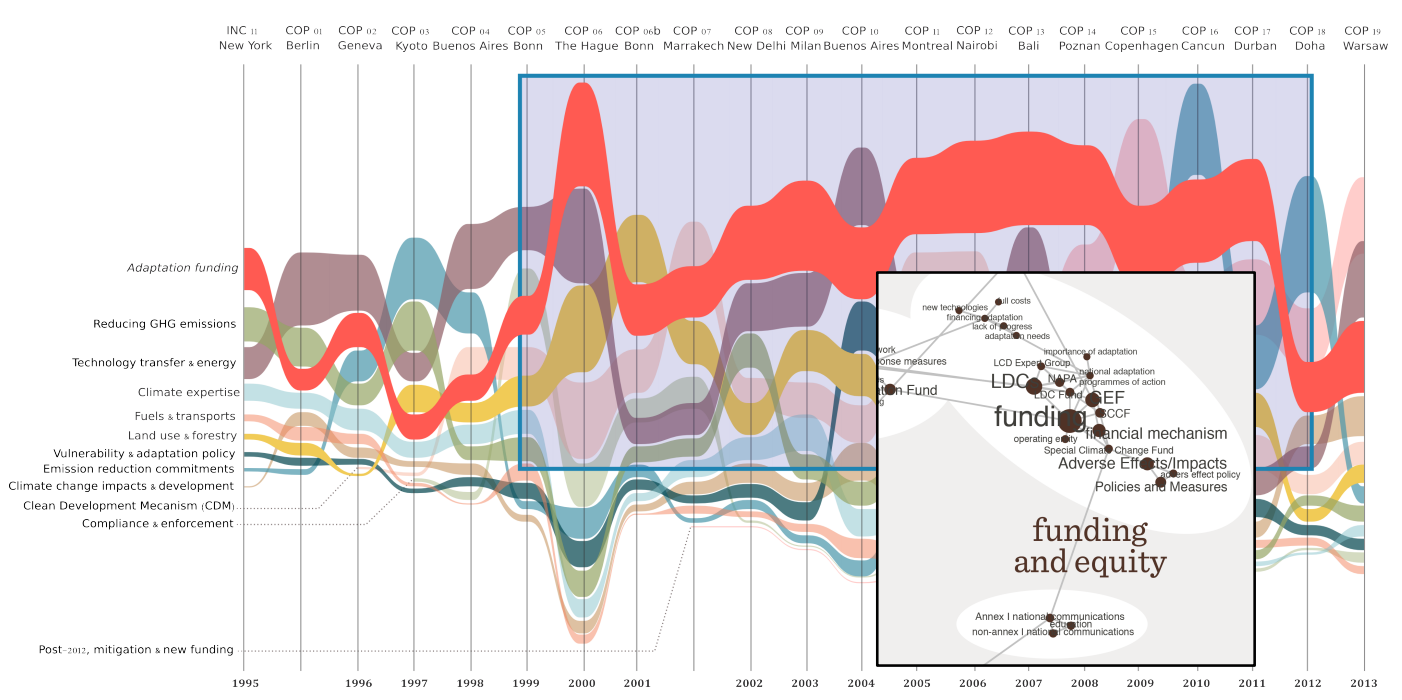

Financement de l'adaptation. Nombre de questions sur le financement, négociées depuis La Haye, sont décidées dans les Accords de Marrakech de 2001 et mises en œuvre par la suite. La publication du troisième rapport d'évaluation du GIEC cette même année conclut avec plus de confiance qu'avant que le changement climatique est dû aux activités humaines, mettant en évidence, de plus, que les impacts se feront sentir, notamment auprès des plus vulnérables, au delà de tout effort d'atténuation du fait de l'inertie du système climatique. Si le chevauchement des questions climatiques et celles de développement des nations est plus clair que jamais, les doutes sur l'efficacité du régime d'atténuation le sont aussi. Le PK est certes sauvé et a pu entrer en vigueur en $2005^{30}$ mais le plus grand émetteur du monde ne l'a pas ratifié. L'AOSIS et le groupe des PMA voient alors s'ouvrir une fenêtre d'opportunité pour pousser la négociation du financement spécifique supplémentaire de l'adaptation (Mace, 2005) (fig. 10). Impératif qui appelle à transformer les façons de penser et de pratiquer le développement et l'aménagement des territoires, l'adaptation commence dès lors

\footnotetext{
${ }^{30}$ Avec l'entrée en vigueur du PK, la COP de Montréal accueille alors, pour la première fois, le sommet des 194 Parties à la Convention (COP11) et celui des 193 parties au Protocole (MOP1, pour meeting of the Parties), les Etats-Unis étant seulement observateurs.
} 
à s'insérer dans des domaines de politiques bien établis, comme celles de l'aide au développement, qu'elle contribue à financer, mais qu'elle menace aussi paradoxalement : d'une part, car on risque de renommer financement de l'adaptation ce qui était déjà financé comme aide officielle au développement, mais encore, d'autre part, car le domaine du développement pose des contraintes parfois contradictoires avec les objectifs de l'adaptation au changement climatique (Magnan, Tubiana et Gemenne, 2010). Après La Haye, donc, trois fonds opérés par le Fonds pour l'environnement mondial (FEM, ou GEF pour Global Environmental Facilty) sont négociés : un fonds spécial du changement climatique (SCCF pour Special Climate Change Fund) destinée à financer des mesures d'atténuation et adaptation (Dessai, 2003) ; un fonds pour les pays les moins avancés (LCD's Fund,) pour le financement des activités d'adaptation, y compris la préparation et la mise en œuvre des programmes action nationaux d'Adaptation (PANA, ou NAPA pour National adaptation programmes of action); et, un fonds d'adaptation. Les décisions de Marrakech à leur égard posent ainsi nombre de questions qui ont été négociées par la suite : mise en œuvre des fonds, adoption de lignes directrices pour la préparation des PANA, et constitution d'un groupe d'experts des PMA dans le cadre de la CCNUCC.

Figure 11. Mécanisme de développement propre, focale sur le pic de 2001

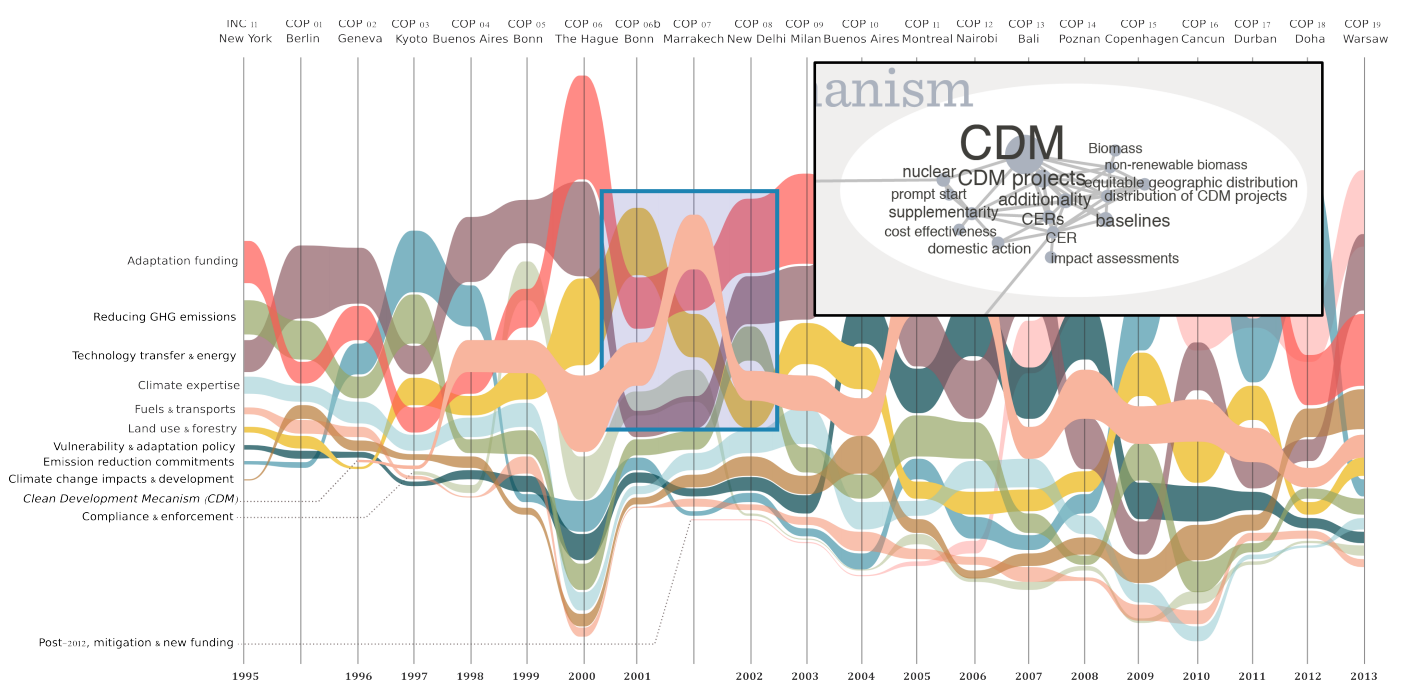

Mécanisme de développement propre. Le Fonds d'adaptation est financé par un prélèvement obligatoire de $2 \%$ sur les réductions d'émissions certifiées 
(CERs) du mécanisme de développement propre (MDP). Et c'est la nation accueillant les projets MDP portés par les entreprises des pays industrialisées, qui doit s'acquitter de cette taxe. Cela implique que le fonds d'adaptation est financé par les PED et non pas sur la base des budgets nationaux des pays industrialisés. Et en effet, ce mécanisme de financement résulte d'un compromis passé entre l'AOSIS et les grands émergents, dont le Brésil, la Chine et l'Inde, qui accueillant le gros des projets $M D P$. Or, tout ceci n'est pas sans poser de problèmes car le fait que l'adaptation se finance en vertu d'une taxe sur les projets bénéfiques d'atténuation est une cause des tensions spécifiques entre adaptation et atténuation. Ce sont les réductions des émissions qui sont pénalisées, non pas les énergies fossiles, dont la taxation était inacceptable pour nombre de pays industrialisés. Le résulate est que l'adaptation se finance au dépens de l'atténuation (Dessai et Schipper, 2003).

Figure 12. Impacts, vulnérabilité et adaptation, et financement de l'adaptation dans le réseau sémantique

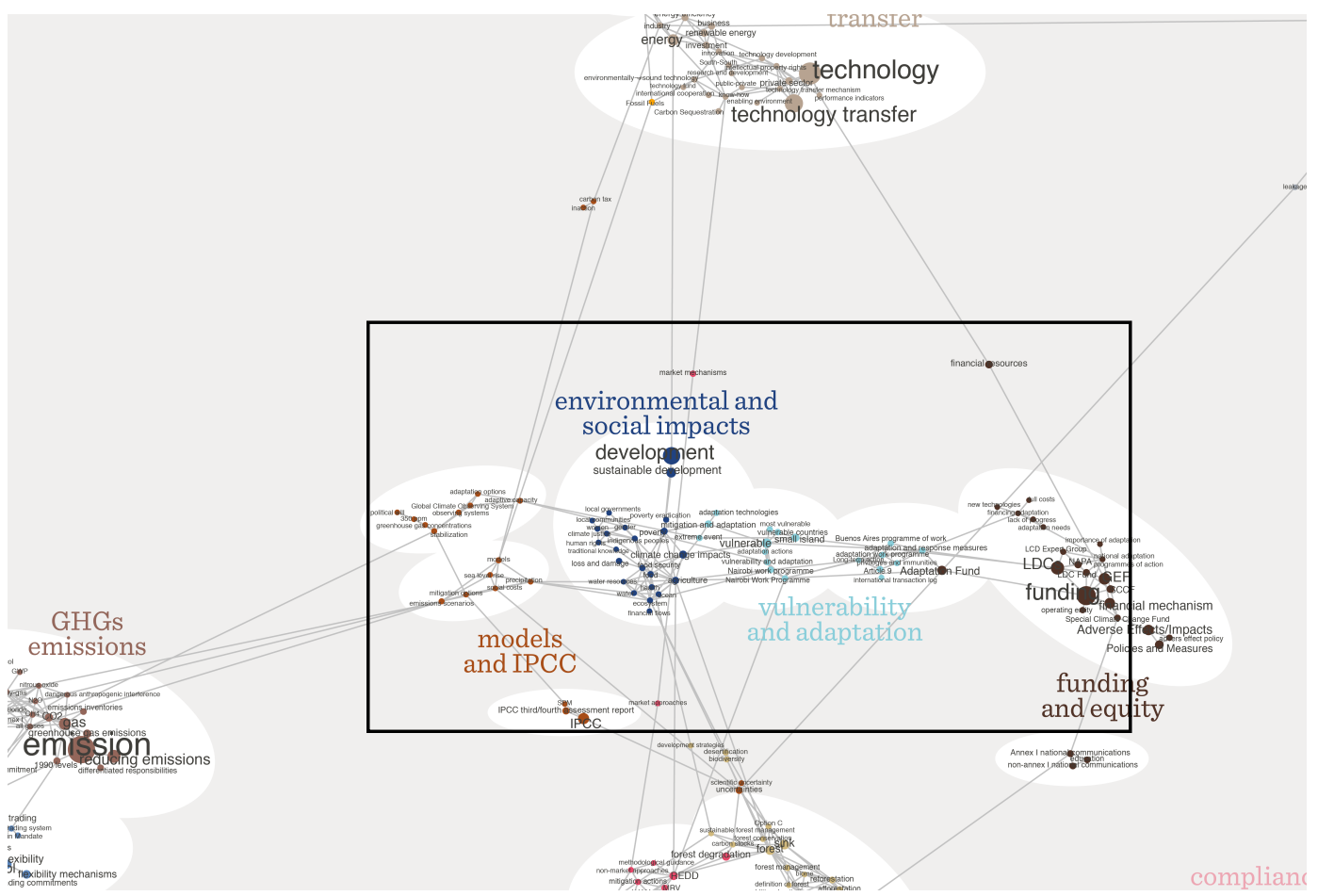

Consolidation du cadrage «impacts, vulnérabilité, et adaptation» et développement dans les discussions. On assiste depuis Marrakech (voir pic 
« adaptation funding \& equity», figure 11) à la consécration progressive d'un nouveau cadrage articulant modèles, impacts, capacités d'adaptation, vulnérabilité, pauvreté, développement (durable), et financement au cœur du régime de la CCNUCC (fig. 12). Lors de la COP8 tenue à Delhi en 2002, l'adaptation apparaît ainsi mise en avant par les organisateurs qui exigent une attention soutenue et une action urgente de la part de tous les pays. L'idée d'un protocole d'adaptation séparé est avancée pour la première fois. La Déclaration ministérielle de Delhi sur les changements climatiques et le développement durable fait de l'adaptation une grande priorité, notamment pour les pays les plus vulnérables (Burton, Huq, Lim, Pilifosova, et al., 2002).

Figure 13. Vulnérabilité et adaptation comme enjeu politique

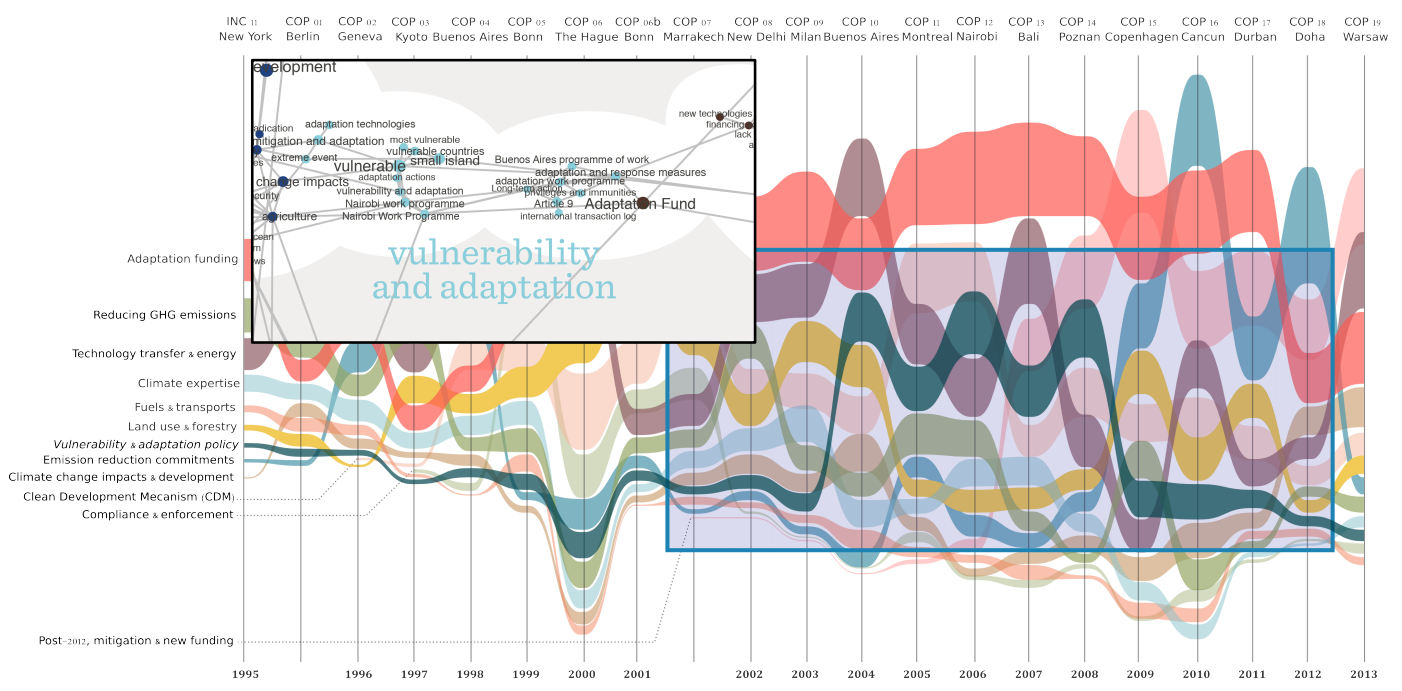

Les négociations insistent de plus en plus sur la nécessité que l'Organe subsidiaire de conseil scientifique et technologique (SBSTA) aborde les aspects scientifiques, techniques, et socio-économiques de la vulnérabilité et de l'adaptation aux changements climatiques (COP9, Milan). C'est alors qu'un nouveau moment de la "traversée du plateau de l'adaptation » commence : celui de l'institutionnalisation (fig. 13). Le Programme de travail sur l'adaptation et sur les mesures d'intervention (COP10, Buenos Aires) définit une approche avec deux voies parallèles : l'une sur les aspects scientifiques, techniques, et socioéconomiques de la vulnérabilité et de l'adaptation dans le cadre du SBSTA; l'autre sur l'amélioration des informations et des méthodologies, des activités 
concrètes d'adaptation, le transfert de technologies d'adaptation et le renforcement des capacités sous l'organe subsidiaire chargé de la mise en œuvre (SBI). Sous le signe de la catastrophe de l'ouragan Katrina à Nouvelle-Orléans, entre autres, on assiste en 2005 à une prise de conscience majeure de la « non invulnérabilité » des pays industrialisés (COP11, Montréal) où l'on continue à discuter les décisions de Buenos Aires. L'organisation la COP12 à Nairobi, Kenya, offre encore une fois l'occasion de lancer un appel, cette fois par le Secrétaire général de l'ONU, Kofi Annan, à une action urgente pour l'adaptation des plus pauvres. Les résultats se manifestent tant sur le plan du financement, avec la structure de gouvernance du Fonds d'adaptation alors presque finalisée, que sur le plan des politiques, avec l'adoption du programme de travail de Nairobi, pour la mise en œuvre de l'adaptation.

Alors, si la position de l'adaptation progresse depuis Marrakech, sa réalisation est pourtant freinée à cause du lien entre « adaptation et effets négatifs des mesures d'intervention » contre le changement climatique (Barnett et Dessai, 2002 ; Khan et Roberts, 2013). La cause se trouve dans les demandes de l'Organisation des pays exportateurs de pétrole (OPEP), fondée sur des dispositions de la Convention, d'une compensation pour les conséquences de la réduction des ventes de pétrole qui pourraient résulter d'une politique efficace d'atténuation au niveau mondial, permettant aux producteurs de pétrole de diversifier leur économie. La conséquence en est une assimilation de l'adaptation des pays pétroliers à celle des pays vulnérables. La controverse, qui résulte des enjeux contradictoires des PMA et producteurs de pétrole, s'éteint lors de la COP13, tenue à Bali, en Indonésie, en 2007, sous le signe du quatrième rapport d'évaluation (AR4) du GIEC. Les conclusions des experts sur la réalité du problème du changement climatique sont plus formelles que jamais quant au besoin d'action tant en matière d'atténuation que d'adaptation à long terme. L'adoption du Plan d'action de Bali inclut parmi ses quatre piliers, l'adaptation, mise dès lors sur pied d'égalité avec l'atténuation, le transfert de technologie et le financement (Pielke, Prins, Rayner et Sarewitz, 2007). L'adaptation aux conséquences du changement climatique est alors inscrite dans le pilier " adaptation»; les réponses aux conséquences des "mesures d'intervention » dans le pilier «atténuation». L'argument des pays pétroliers tombe. Les PMA vulnérables voient dès lors la voie libre pour négocier la gouvernance du financement des besoins d'adaptation sans ambigüité. Les négociations sur ces questions se terminent à Poznań, en 2008.

2.3. Le financement promis à l'aune du nouveau régime d'atténuation (2009présent) 
En 2009, l'espace thématique se restructure à nouveau autour d'une échéance clé, marquant un avant et un après dans le régime climatique : la COP 15 qui se tient à Copenhague en décembre 2009. L'enjeu en est la définition d'un régime d'engagements post-Kyoto à l'aune d'un nouveau cadre stratégique, mais aussi des mécanismes de financement de l'adaptation, utilisées comme monnaie d'échange pour engager les PED dans la négociation.

Figure 14. Nouveau cadre stratégique post-2012 (y compris débats sur le nouveau Fonds vert pour le climat)

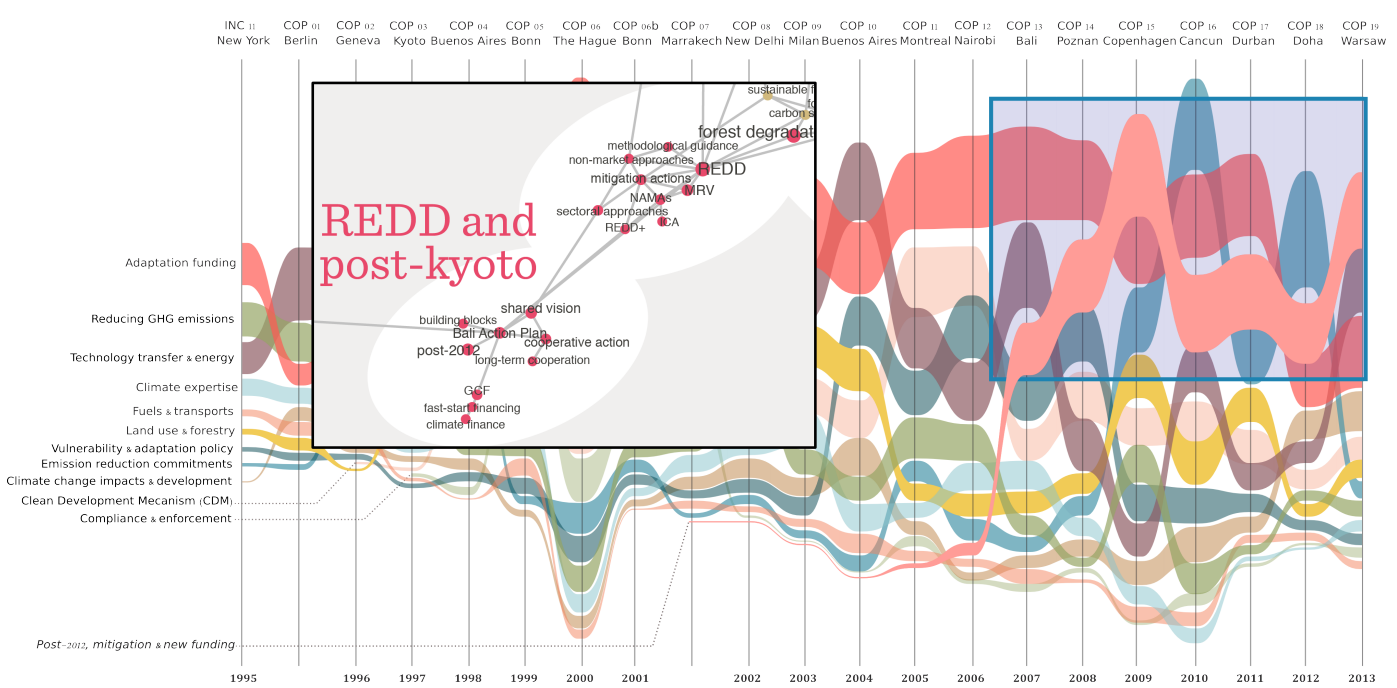

Vers un nouveau cadre post-Kyoto. La préparation de la renégociation du PK pour la deuxième période d'engagement (en vertu de l'article $9 d u P K$ ) se pose dès Buenos Aires (2004), mais se fait essentiellement à Bali $(C O P 13,2007)$ et à Poznań (COP14, 2008) (Dahan, 2009) (figure 14). Le Plan d'action de Bali pose les piliers d'un nouveau cadre stratégique des négociations sans définir le contenu politique de l'accord post-2012 ${ }^{31}$. A l'issue de la conférence, deux voies de négociations distinctes avaient été établies dans la feuille de route de Bali. La première est celle des négociations sur la vision commune de la Coopération à

\footnotetext{
${ }^{31}$ Le mécanisme de Réduction des émissions liées à la déforestation et la dégradation forestière (REDD, et REDD+), ainsi que la possibilité des accords sectoriels pour la réduction des émissions industrielles (reflétant l'enjeu de l'intégration des politiques climatiques) sont deux autres aboutissements importants des négociations de Bali, dont les termes configurent ce cluster.
} 
long terme réunissant toutes parties à la Convention. La seconde concerne la définition d'une deuxième période d'engagement post-2012 sur le PK qui regroupe ses 193 Etats parties ${ }^{32}$. Cette approche à deux voies a été opposée par des Parties proposant une seule voie de négociation pour aboutir à un nouvel instrument intégrant les grands pays émergents, responsables d'une partie considérable des émissions au cours des années 2000 et qui ne sont soumis à aucune contrainte au titre du PK. Un nouvel accord devrait venir, de ce fait, changer cette situation, en faisant participer à l'effort non plus les seuls responsables historiques mais plutôt l'ensemble des responsables des émissions. C'est donc le principe d'équité de la répartition des efforts de réduction entre les Etats qui dominait les négociations jusque là qui est remis en question (Brunnée et Streck, 2013).

La COP15 réunit près de 120 dirigeants du monde afin d'accoucher d'un nouvel accord post-2012. Les divisions entre les pays qui souhaitent des mesures énergiques et ceux qui souhaitent différer tout engagement contraignant sont aussi grandes que les attentes. Les négociations étant dans l'impasse, l'échec est majeur (Aykut et Dahan, 2011). L'Accord de Copenhague est un texte de 3 pages rédigé à huis clos par une vingtaine de chefs d'Etat, sous l'égide des États-Unis et les grands émergents afin de sauver la COP du naufrage total. S'il établit certes l'objectif de limiter la hausse moyenne des températures à $2^{\circ} \mathrm{C}$ par rapport au niveau préindustriel que les scientifiques du GIEC avaient estimé comme relativement sûre pour éviter un «changement climatique dangereux», l'accord adopté n'est cependant pas contraignant. Les réductions proposées par les pays développés et les actions visant à limiter la croissance des émissions proposées par les PED sont inscrites dans des appendices à l'Accord et doivent être révisées itérativement, jusqu'à ce que la somme des engagements permette d'atteindre l'objectif global. Cette nouvelle approche bottom-up, assimile l'équité aux bonnes intentions des Etats en fonction des objectifs qu'ils acceptent de se fixer (Dahan, 2014). Or, le total de ces engagements est bien en deçà de ce que l'on devait obtenir en fonction de l'objectif des $2^{\circ} \mathrm{C}$. Ce sont là les nouvelles règles du jeu donnant le ton à la suite de la négociation. Les PED dont les appuis sont plus nécessaires que jamais pour faire adopter le texte sont inquiets. Il faut alors les convaincre de signer. Mais, quel intérêt ont-ils à coopérer ? De grandes déclarations sur l'urgence de l'action en matière d'adaptation, explicitement associées à la réduction de la vulnérabilité et le renforcement de la résilience sont énoncées dans l'accord, appuyées par 30 milliards de dollars en «financement de

\footnotetext{
${ }^{32}$ Chacune des ces voies de négociations à donné lieu à un groupes de travail ad hoc : l'un sur l'action coopérative à long terme (l'AWG-LCA) et l'autre sur le Protocole de Kyoto (AWG-KP).
} 
démarrage rapide » pour les PED au cours de 2010-2012 et un "élargissement » à 100 milliards de dollars par an jusqu'en 2020, dont une bonne partie serait canalisée à travers un nouveau mécanisme créé au milieu de l'échec: le Fonds vert pour le climat. Aucune règle précise à son égard n'ayant été fixée, il devient alors un nouvel objet de débats. Alors que les pays développés défendent un engagement fort en ce sens, les ONG et certains pays vulnérables n'y voient qu'un recyclage d'aides existantes pour le développement (Klein, 2014) (Gemenne, 2013a).

Figure 15. Le retour de l'atténuation : engagements de réduction post 2012 (y

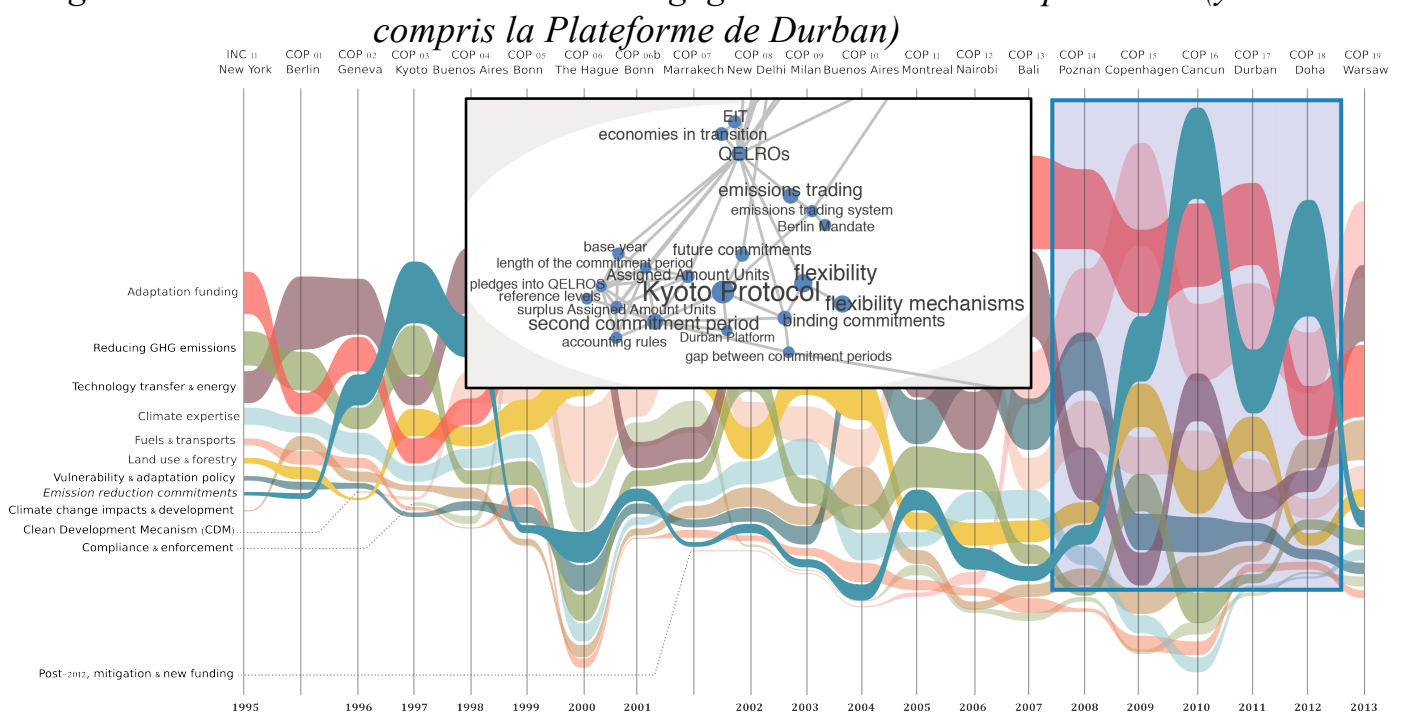

Le financement de l'adaptation et des pertes et dommages, des éléments de complexité supplémentaires. Le sérieux des engagements est mis à l'épreuve entre Cancun (COP16, 2010) et Varsovie (COP19, 2013). D'abord, en matière d'atténuation (voir figure 15), l'objectif des $2^{\circ} \mathrm{C}$ devient officiel à partir de Cancun. Or, la définition du futur accord restera une question pendante, tout comme la nature des engagements des Etats-Unis et des pays émergents. C'est dans ce dessein qu'est mise sur pied la Plateforme de Durban : un ensemble de décisions, comprenant la création d'un groupe de travail ad hoc, établi en vue d'aboutir en 2015, lors de la COP21 de Paris, à un nouveau régime climatique post Kyoto, celui-ci devant inclure, contrairement au PK, l'ensemble de la communauté internationale dans la lutte contre le changement climatique. Les Accords de Cancun établissent également que l'adaptation soit désormais abordée 
avec le même niveau de priorité que l'atténuation. Cette exigence se traduit par la création et la mise en œuvre d'un comité d'adaptation. Composé de 16 membres, son but est d'élaborer un plan sur 3 ans visant à faciliter une action renforcée, instituer un cadre d'adaptation et mettre en œuvre des plans nationaux d'adaptation (PAN). Enfin, en matière de financement, les règles du nouveau Fonds vert pour le climat sont mises en œuvre à Doha avec un comité permanent des finances, placé directement sous la tutelle de la Conférence, chargé de sa surveillance. Son pouvoir et sa portée sont cependant très contestés, certains pays souhaitant limiter drastiquement ses capacités en matière de décision de financement. A ceci s'ajoute la nouvelle question des "pertes et dommages" causés par les impacts négatifs du changement climatique, concept aux termes controversés, impliquant la responsabilité des pays industrialisés, qui se traduit en un mécanisme international sur les pertes et dommages mise en place à Varsovie, à l'aune d'une montée en puissances des questions relatives aux phénomènes météorologiques extrêmes (fig. 9) dont la réalité est attestée le rapport spécial du GIEC de 2012 (dit SREX) (IPCC, 2012).

Si l'adaptation semble être installée dans le régime de la CCNUCC, sa place dans le régime climatique à venir reste à définir, notamment en ce qui concerne un objectif global d'adaptation pour la sécurité humaine dans un contexte d'augmentation des températures dans le monde d'au moins $+2^{\circ} \mathrm{C}$ (Magnan, Ribera, Treyer et Spencer, 2014). Le défi est grand: dans les circonstances actuelles, le processus de la CCNUCC fonctionne toujours comme un monde à part. Nombre de questions se posent quant à l'efficacité et, in fine, la réforme de ce processus, les émissions de carbone continuant d'augmenter. C'est ainsi que S. Aykut et A. Dahan ont récemment parlé avec justesse d'un "schisme de réalité » : entre celle, extérieure d'un monde de la globalisation des marchés, de l'exploitation effrénée des ressources d'énergies fossiles et des Etats pris dans une concurrence économique féroce, et celle, intérieure, d'une négociation caractérisée par son immobilisme, sans prise sur le réel, et ce malgré le discours scientifique qui accentue l'impératif d'une action centralisée globale (Aykut et Dahan, 2015). L'institutionnalisation de l'adaptation et de son financement, on pourrait l'espérer, devrait mettre le processus onusien plus en prise avec certains enjeux des réalités globales : celle d'un changement climatique inéluctable et dont les impacts se font sentir. La question se pose, cependant, de savoir dans quelle mesure elle ne conduit paradoxalement à une saturation majeure du processus en le chargeant d'une complexité spécifique supplémentaire. Dans une arène à vocation spécialisée dont la capacité de charge est limitée, cela met à l'épreuve la Convention dans un temps où le multilatéralisme est en échec. La question reste ouverte de savoir si la CCNUCC est à même de traiter la question de l'adaptation à l'échelle globale ? 


\section{Des visualisations et des narrations à leur intégration dans une plateforme}

\section{en ligne : perspectives futures}

Nous avons commencé cet article en constatant combien la durée des négociations et la multiplication concomitante de questions concourantes constituent deux sources de complexité qui rendent les processus difficile à saisir dans son ensemble pour toute personne s'y intéressant ou y participant. Nous avons alors posé la question de savoir comment l'exploitation de méthodes numériques combinant des approches quantitatives et qualitatives peut contribuer à maîtriser cette complexité et, in fine, aider à comprendre la trajectoire controversée de l'adaptation dans un régime dominé par l'objectif d'atténuation. Si les sources textuelles sur les négociations (récits journalistiques, analyses scientifiques fines et documents originaux du processus de négociations) abondent, on ne peut pas dire qu'il en soit de même des représentations graphiques alors même que les changements d'échelle qu'elles offrent ouvrent de nouvelles perspectives quant au déploiement thématique des COP. C'est là un manque que nous avons cherché à combler par les visualisations de données BTN qui peuvent être un supplément précieux aux histoires et analyses existantes, soit parce qu'elles les confirment, soit parce qu'elles les infirment.

En offrant une "lecture distante ( Moretti, 2013) des négociations, nos cartes ont permis de produire un récit qui explique l'inflation thématique entre les sessions de Berlin 1995 et de Varsovie 2013; inflation caractérisé par l'institutionnalisation de la notion d'adaptation qui s'impose au milieu d'un régime d'atténuation de plus en plus éclaté. Alors que le réseau sémantique nous a permis d'identifier les principales thématiques mobilisées durant les négociations, le diagramme de flux permet de suivre dans le temps les cadres mobilisés par les acteurs. En nous focalisant sur le partage entre atténuation et adaptation, la perspective diachronique qu'offre cette visualisation a permis d'identifier trois grandes périodes dans les négociations délimitées par la COP 6 (La Haye) et la COP 15 (Copenhague). La lecture du graphe de flux montre combien la question de l'adaptation et de son financement a toujours été un objet de négociations. Même si l'accent est essentiellement mis sur l'objectif de réduction des émissions de GES, le financement de l'adaptation fait partie des thèmes majeurs dès les années 90 . Les actions en matière d'adaptation restent, cependant, un sujet minoritaire jusqu'en 2004. Ce n'est que depuis la crise des négociations achoppant sur la mise en ouvre du Protocole de Kyoto à La Haye, en 2000, que le thème du financement de l'adaptation, puis celui de l'action d'adaptation, deviennent officiellement très importants, au moins jusqu'en 2009, lorsque la COP de Copenhague et la définition de nouveaux engagements d'atténuation regagneront le haut de l'agenda. Le financement de l'adaptation 
s'impose comme le thème principal à la Haye (2000). Parallèlement, et dès 2001 avec la COP de Marrakech, on observe l'avènement d'un nouveau cadrage qui articule impacts, adaptation, vulnérabilité et développement. Cette dimension politique de l'adaptation devient extrêmement prégnante à compter de la COP de Buenos Aires en 2004. La montée en puissance de ces deux dimensions (financières et politiques) explique le haut plateau de l'adaptation que nous observons clairement sur notre graphe. La dernière période des négociations est caractérisée par une reconfiguration importante des thématiques. La renégociation $\mathrm{du}$ Protocole de Kyoto entraine un rééquilibrage des thématiques. En s'institutionnalisant, le financement de l'adaptation et encore des pertes et dommages ajoutent de fait une nouvelle source de complexité qu'il faut intégrer dans le régime de la CCNUCC ; régime dont les fondements juridiques (principe de responsabilités communes et différentiés) font à ce jour l'objet d'une remise en question importante. C'est de l'issue de ces discussions dont dépendra le nouvel accord qui devrait être adopté à la COP21 de Paris 2015. Ainsi, Si le débat atténuation vs adaptation semble dépassé, la question persiste de savoir quelle combinaison de mesures relevant d'une stratégie et de l'autre privilégier et comment les articuler au sein de la CCNUCC, compte tenu des ressources et capacités limitées.

Plusieurs autres récits sont possibles pour rendre compte de l'évolution ou de l'articulation de chaque thématique à chaque COP. On peut dès lors, en composant visualisations et narration, redire autrement la complexité des négociations, en faire des constatations ou formuler de nouvelles hypothèses appelant de nouvelles recherches. Des pistes intéressantes s'ouvrent, les visualisations pouvant être refaites sur la base de nouvelles sélections de termes et en testant d'autres calibrations des algorithmes afin de tester ces hypothèses. En outre, on peut également penser à traiter les sessions des organes subsidiaires de la $\mathrm{COP}$, ou à raffiner les méthodes pour faire émerger les lignes argumentatives et les structures et modalités d'énonciation autour de thèmes spécifiques. Et enfin, il restent encore plusieurs volumes de l'archive BTN sur les processus de négociation en matière d'environnement avec lesquelles de nouvelles expérimentations pourraient être menées.

Comme les retours que nous avons obtenus auprès des négociateurs nous l'indiquent, l'utilisation des cartes présentées dans cet article suscite des attentes, notamment dans le cadre la préparation de la COP21, à Paris, en 2015. En ce sens, la prochaine étape de notre expérience est d'articuler ces cartes ainsi que d'autres visualisations du processus de négociation au sein d'un site web, qui devrait voir le jour début 2015. Grâce à une interface interactive, il s'agit de permettre une exploration des cartographies et des données qui les fondent. $\mathrm{La}$ plateforme web sur la trajectoire d'institutionnalisation du thème de l'adaptation 
se structure en plusieurs volets " arènes », comprenant la CCNUCC, le GIEC, les publications scientifiques, les discours media, et un «web doc» interactif sur l'adaptation dans le bassin de la Garonne ${ }^{33}$. Chacun de ces volets articule des données, des visualisations de données et des narrations combinant, une approche "author-driven" typique d'une analyse scientifique, ou "readerdriven " plus adaptée à des dispositifs d'exploration de données (Segel et Heer, 2010). De plus les visualisations en ligne permettent un interactivité permettant d'affiner/élargir la visualisation via les fonctionnalité de «zoom in » et «zoom out » (par exemple pour le réseau sémantique) ou de renvoyer directement aux données source, à un glossaire critique, et à une chronologie générale de la trajectoire de l'adaptation entre science et politique. Un évènement de lancement et une stratégie de communication assurent la dissémination du site dans le cadre de la préparation de la COP21. C'est à cette fin que le protocole de production des graphes comprend la construction, dès le début, d'une communauté d'utilisateurs potentiels des visualisation afin d'obtenir des retours d'expérience. Comment ces cartographies seront-elles mobilisées pour des utilisations scientifiques ou pour l'action publique, c'est là une question pendante qui va guider la dernière étape de notre expérience : quelles trajectoires vont suivre ces visualisations une fois sorties du laboratoire?

\section{Références}

ARISTOTE (2002), Métaphysique, tome 2 livre H-N, Paris, Librairie Philosophique Vrin.

AYKUT S. et DAHAN A. (2011), «Le régime climatique avant et après Copenhague: sciences, politiques et l'objectif des deux degrés» », Natures Sciences Sociétés, vol. 19, n² 2, p. 144-157.

AYKUT S. (2012), Comment gouverner un'nouveau risque mondial'? La construction du changement climatique comme problème public à l'échelle globale, européenne, en France et en Allemagne, these de doctorat, Ecole des Hautes Etudes en Sciences Sociales (EHESS).

AYKUT S. et DAHAN A. (2015), «La gouvernance du changement climatique. Anatomie d'un schisme de réalité », Le Gouvernement des technosciences : gouverner le progrès et ses dégâts depuis 1945, in Pestre D. (éd.), Paris, La Découverte.

\footnotetext{
${ }^{33}$ Des informations plus détaillées sur la plateforme et ses fonctionnalités, ainsi qu'un lien vers la plateforme dès qu'elle sera en ligne sont disponibles à l'adresse suivante: http://www.medialab.sciences-po.fr/fr/projets/medea-mapping-environmentaldebates-on-adaptation/
} 
BARATS C. (2013), Manuel d'analyse du web en Sciences Humaines et Sociales, Armand Colin.

BARNETT J. et DESSAI S. (2002), « Articles 4.8 and 4.9 of the UNFCCC: adverse effects and the impacts of response measures », in Climate Policy, vol. 2, $\mathrm{n}^{\circ} 2$, p. 231-239.

BAYA-LAFFITE N. et VENTURINI T. (2014), « What digital mapping reveals about debates on climate change adaptation », in Climate exchanges: Language, Media and Politics, Centre franco-norvégien en sciences sociales et humaines, Paris.

BERRANG-FORD L. et al. (2014), « What drives national adaptation? A global assessment »,. Climatic Change, p. 1-10.

BETZOLD C., CASTRO P. et WEILER F. (2012), « AOSIS in the UNFCCC negotiations: from unity to fragmentation? », in Climate Policy, vol. 12, $\mathrm{n}^{\circ} 5, \mathrm{p}$. 591-613.

BLONDEL V.D., GUILLAUME J-L, LAMBIOTTE R. et LEFEBVRE E. (2008), « Fast unfolding of communities in large networks », in Journal of Statistical Mechanics: Theory and Experiment, vol. 2008, $\mathrm{n}^{\circ}$ 10, p. P10008.

BRUNNÉE J. et STRECK C. (2013), «The UNFCCC as a negotiation forum: towards common but more differentiated responsibilities $»$, . Climate Policy, vol. $13, \mathrm{n}^{\circ} 5$, p. 589-607.

BURTON I., HUQ S., LIM B., PILIFOSOVA O. et al., 2002, «From impacts assessment to adaptation priorities: the shaping of adaptation policy », in Climate policy, vol. 2 , n ${ }^{\circ}$ 2, p. 145-159.

COLLINS H. (1992), Changing Order: Replication and Induction in Scientific Practice, University Of Chicago Press

COLLINS H. et PINCH T. (2001), Tout ce que vous devriez savoir sur la science, Seuil.

DAHAN A. (2009), « Entre Poznan et Copenhague: le régime climatique au milieu du gué », in Natures Sciences Sociétés, vol. 17, n 3, p. 271-282.

DAHAN A. (2014), «L'impasse de la gouvernance climatique globale depuis vingt ans. Pour un autre ordre de gouvernementalité », in Critique internationale, vol. , n 1 , p. 21-37.

DAHAN A. (2010), « Putting the Earth System in a numerical box? The evolution from climate modeling toward global change », in Studies in History and Philosophy of Science Part B: Studies in History and Philosophy of Modern Physics, vol. 41, n 3, p. 282-292. 
DAHAN A. et AYKUT S. (2012), De Rio 1992 à Rio 2012 vingt annees de negociations climatiques: quel bilan? Quel role pour l'europe? Quels futurs?, Rapport pour le Centre d'analyse stratégique, Paris, CAS.

DESSAI S., 2003, « The special climate change fund: Origins and prioritisation assessment », in Climate Policy, vol. 3, n 3, p. 295-302.

DESSAI S. et SCHIPPER E.L., 2003, « The Marrakech accords to the Kyoto protocol: Analysis and future prospects »,. Global Environmental Change, vol. $13, \mathrm{n}^{\circ} 2$, p. 149-153.

DESSAI S., LACASTA N.S. et VINCENT K. (2003), « International Political History of the Kyoto Protocol: from The Hague to Marrakech and Beyond », in International Review for Environmental Strategies, vol. 4, n 2, p. 183-205.

EWICK P. et SILBEY S.S., 1995, « Subversive Stories and Hegemonic Tales: Toward a Sociology of Narrative », in Law \& Society Review, vol. 29, n², p. 197-226.

GEMENNE F. (2013a), «Focusing global adaptation funding to the most vulnerable », Adaptation au changement climatique - cadrages et connaissances en débat Adaptation to climate change - framings and knowledge in debate. Atelier international ANR MEDEA \& ClimaConf, 29-05-2013, Paris, Sciences Po.

GEMENNE F. 2013b, « Les négociations internationales sur le climat. Une histoire sans fin? », in Négociations internationals, Petiteville, F. et Placidi-Frot D. (éds) p. 395-422.

GODARD O., (2010), « Cette ambiguë adaptation au changement climatique », in Natures Sciences Sociétés, vol. 18, n 3, p. 287-297.

HARLEY J. B. (2002), The New Nature of Maps: Essays in the History of Cartography, JHU Press.

HUQ S. et REID H. (2004), « Mainstreaming adaptation in development », in IDS bulletin, vol. 35, $\mathrm{n}^{\circ} 3, \mathrm{p} .15-21$.

HUTCHBY I. (2001), « Technologies, Texts and Affordances », in Sociology, vol. $35, n^{\circ} 2$, p. $441-456$.

IISD (2013a), Manual and Style Guide, New York, International Institute for Sustainable Development - Reporting Services.

IISD, 2013b, « Warsaw Climate Change Conference (COP19) 11-22 November $2013 »$, . Earth Negotiations Bulletin, vol. 12, n 588.

IPCC (2012), Managing the Risks of Extreme Events and Disasters to Advance Climate Change Adaptation. A Special Report of Working Groups I and II of the 
Intergovernmental Panel on Climate, Cambridge, UK, and New York, NY, USA, Cambridge University Press.

JACOMY M., VENTURINI T., HEYMANN S. et BASTIAN Mat, 2014, «ForceAtlas2, a Continuous Graph Layout Algorithm for Handy Network Visualization Designed for the Gephi Software », in PLOS ONE, vol. 9, n 6, p. e98679.

KHAN M.R. et ROBERTS J.T., 2013, « Adaptation and international climate policy », Wiley Interdisciplinary Reviews: Climate Change, vol. $4, \mathrm{n}^{\circ} 3, \mathrm{p}$. 171-189.

KLEIN R., 2014, « Negotiating Adaptation to Climate Change under the UNFCCC: Areas of Controversy », in EMAPS Sprint on Climate negotiations, SciencesPo, Paris.

LATOUR B. (1993) «Le pédofil de Boavista, montage photo-philosophique », in Petites leçons de sociologie des sciences, p. 171-225.

LATOUR B. (1987), «Les“vues” de l'esprit», in Réseaux, vol. 5, n 27, p. 7996.

MACE M.J. (2005), «Funding for Adaptation to Climate Change: UNFCCC and GEF Developments since COP-7 », in Review of European Community \& International Environmental Law, vol. 14, n ${ }^{\circ} 3$, p. 225-246.

MAGNAN A., RIBERA T., TREYER S. et SPENCER (2014), « What adaptation chapter in the New Climate Agreement? », Iddri Policy Briefs, vol. 2014, n 9.

MAGNAN A., TUBIANA L., et GEMENNE F. (2010), Anticiper pour s'adapter: Le nouvel enjeu du changement climatique, Pearson.

MANN M. (2013), The Hockey Stick and the Climate Wars: Dispatches from the Front Lines, Columbia University Press.

MONMONIER M. S., 1995, Drawing the Line: Tales of Maps and Cartocontroversy, Henry Holt \& Co.

MORETTI F. Franco, 2013, Distant Reading, Verso Books.

PIELKE Jr. R.A. (2005), « Misdefining “climate change": consequences for science and action », in Environmental Science \& Policy, vol. 8, n 6, p. 548-561.

PIELKE Jr. R.A., PRINS G., RAYNER S. ET SAREWITZ D. (2007), « Climate change 2007: Lifting the taboo on adaptation », in Nature, vol. $445, n^{\circ} 7128$, p. 597-598.

PIELKE Jr. R.A, 1998, « Rethinking the role of adaptation in climate policy »,. Global Environmental Change, vol. 8, $\mathrm{n}^{\circ}$ 2, p. 159-170. 
PLANTIN J-C. (2013), « D’une carte à l'autre: Le potentiel heuristique de la comparaison entre graphe du web et carte géographique », Manuel d'analyse du web en Sciences Humaines et Sociales, Barats C. (éd.), Armand Colin, p. 229-245.

PLATON, (1976), Oeuvres, tome 8, 2e partie: Théétète, Édition : 1. A. Diès éd. Paris, Belles Lettres.

SCHIPPER L., 2006, " Conceptual History of Adaptation in the UNFCCC Process ",. Review of European Community \& International Environmental Law, vol. 15, n 1, p. 82-92.

SEGEL E. et HEER J. (2010), « Narrative visualization: Telling stories with data ", in Visualization and Computer Graphics, IEEE Transactions on, vol. 16, $\mathrm{n}^{\circ} 6$, p. 1139-1148.

Turnbull David, 2000, Masons, Tricksters and Cartographers: Comparative Studies in the Sociology of Scientific and Indigenous Knowledge, Psychology Press.

VENTURINI T., 2012, « Building on faults: How to represent controversies with digital methods »,. Public Understanding of Science, vol. 21, n 7, p. 796-812.

VENTURINI T., RICCI D, MAURI M., KIMBELL L, et al. (Forthcoming in 2015), « Designing Controversies and their Publics », in Design Issues

VENTURINI T. (2010), « Diving in magma: how to explore controversies with actor-network theory », in Public Understanding of Science, vol. 19, $\mathrm{n}^{\circ} 3$, $\mathrm{p}$. 258-273.

VENTURINI T, BAYA-LAFFITE N. COINTET J-P., GRAY I., ZABBAN V. et PRYCK K. 2014, « Three maps and three misunderstandings: A digital mapping of climate diplomacy », in Big Data \& Society, vol. 1, n 2.

WEEDS J. et WEIR D. (2005), « Co-occurrence retrieval: A flexible framework for lexical distributional similarity », in Computational Linguistics, vol. 31, $\mathrm{n}^{\circ} 4$, p. 439-475.

YAMIN F. (2014), «Adaptation Negotiations History », in EMAPS Sprint on Climate negotiations SciencesPo, Paris.

YAMIN F. (2005), « The European Union and future climate policy: Is mainstreaming adaptation a distraction or part of the solution? », in Climate Policy, vol. 5, n 3, p. 349-361.

YAU N. (2013), Data visualisation: De l'extraction des données à leur représentation graphique, Editions Eyrolles. 


\section{Financement}

Cet article présente une partie des résultats du projet MEDEA (Mapping Envrionmental DEbates on Adaptation) financé par l'ANR dans le cadre du programme 'Changements Environnementaux Planétaires et Sociétés' de 2011 (ANR-11-CEPL-0004). Les auteurs tiennent à remercier les contributions faites lors des « Sprints » organisés dans le cadre du projet EMAPS (Electronic Maps to Assist Public Science) financé par le 7ème Programme Cadre de l'Union Européenne, ainsi que les commentaires reçus à l'occasion de nombreuses présentations orales de ces travaux, et notamment lors de la Conférence internationale « New perspectives on global environmental images » organisé par le GIS Climat et l'Ademe en octobre 2014. Les auteurs déclarent n'avoir aucun conflit d'intérêt. 